\title{
Gut Microbes and Microbial Metabolites in Colorectal Cancer Complicated with Different Serum Albumin Levels
}

\section{Shuwen Han}

Huzhou Central Hospital

\section{Wei Wu}

Huzhou Central Hospital

\author{
Miao Da \\ huzhou Third Municipal Hospital
}

\section{Qing Zhou}

Huzhou Central Hospital

\section{Jiamin Xu}

Huzhou University

Jing Zhuang

Huzhou University

Jin Liu

Huzhou Central Hospital

Xi Yang ( $\square$ yangxi0601@hotmail.com )

Huzhou Central Hospital

\section{Research article}

Keywords: Bacteria, fungus, Metabolites, Albumin, Colorectal Cancer

Posted Date: January 13th, 2020

DOI: https://doi.org/10.21203/rs.2.20754/v1

License: (1) (1) This work is licensed under a Creative Commons Attribution 4.0 International License. Read Full License 


\section{Abstract}

Background: Patients with colorectal cancer (CRC) are at risk of malnutrition. Gut microbes and microbial metabolites are involved in the initiation and development of CRC.

Purpose: To investigate serum protein levels in CRC patients and explore the role of gut microbes and microbial metabolites in CRCs complicated with different serum albumin levels.

Methods: Overall, 398 CRC patients and same number of healthy volunteers in Huzhou Central Hospital from January 2016 to December 2018 were recruited to compare serum protein levels. The serological indicators were detected by Abbott Automatic Biochemical Analyzer(HCHL-YQ-SH-01). A total of 30 and 56 stool samples from CRC patients were used to detect intestinal microbes and microbial metabolites, respectively. Bacterial 16S V3-V4 and fungal ITS ribosomal DNA genes were sequenced and gas chromatography/mass spectrometry (GC/MS) was performed to detect microbial metabolites.

Results: Some serum protein-related indicators in the CRC group were lower than those in the control group ( $\mathrm{p} \otimes 0.05$ ). The total protein and albumin levels in colon cancer patients were lower than those in

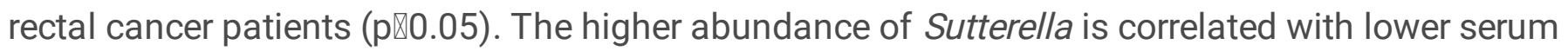
albumin level in CRCs. There were statistically significant differences in the abundance of fungi including

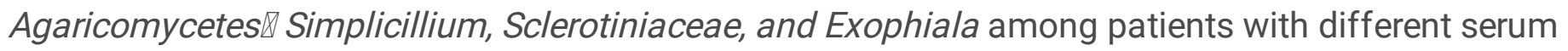
albumin levels. Multiple gut bacteria and fungi are closely related to serum albumin levels. We found some characteristic microbial metabolites in CRCs complicated with different serum protein levels.

Conclusions: The different serum albumin levels were associated with the gut microbes and microbial metabolites in CRCs. It may provide novel ideas for basic research and clinical application.

\section{Introduction}

Colorectal cancer (CRC) is one of the most common malignancies and the incidence of CRC has increased in recent years ${ }^{[1]}$. With advances in multidisciplinary comprehensive treatment (MDT), molecular targeting treatment and immunotherapy, the 5-year survival time and 10-year survival time of CRC patients has reached over $30 \%$ and $20 \%$, respectively ${ }^{[2-4]}$. CRC is a disease with insidious pathogenesis, with patients often experiencing a series of pathophysiological changes such as inflammation, ulcers, hemorrhage, and intestinal obstruction that inhibits the absorption of nutrients by the body. CRC cells require a large amount of nutritional support in the rapid proliferation phase resulting in the depletion of nutrients in the body. Therefore, CRC patients are at risk of malnutrition at an early stage of the disease. Malnutrition has adverse effects on the choice of surgical timing, postoperative recovery, immune function, incidence of complications, and hospitalization costs. Therefore, the early risk assessment of malnutrition is more beneficial than intervention after the occurrence of malnutrition.

Many studies supported that gut microbes ${ }^{[5-7]}$ and microbial metabolites ${ }^{[8,9]}$ are involved in the occurrence and development of CRC. The main clinical symptoms of malnutrition include fatigue, 
emaciation, and edema, and it is caused by the consumption of carbohydrates, fats, and proteins. We have systematically summarized the association network between the gut microbes and metabolism of nutrients including carbohydrates, fat and protein in the form of literature review ${ }^{[10]}$. Previous studies have found that CRCs are often complicated with dyslipidosis and there were differences in gut bacteria among CRC patients with different lipid metabolism levels ${ }^{[11]}$. However, the correlation between intestinal microecological environment and protein levels in CRC patients remains to be confirmed.

Many studies have reported that high-protein diets leaded to changes in gut microbes and microbial metabolites. For instance, a dog model showed that high-protein diet increased the abundance and activity of butyrate-producing bacteria in stool samples ${ }^{[12]}$. Jianping, et al. reported that the high-protein diet increases the abundance of Bacteroidetes, Prevotella, Oscillospira and Sutterella bacterial species, and decreases the abundance of Firmicutes ${ }^{[13]}$. Chunlong, et al. reported that high-protein diet can decrease the numbers and activity of propionate- and butyrate-producing bacteria ${ }^{[14]}$. Xiangyu, et al. reported that high-protein diet could cause an increase in protein fermentation and lead to increased production of detrimental metabolites ${ }^{[15]}$. Carlo, et al. reported that high-protein diet can increase the concentration of ammonia in canine feces ${ }^{[16]}$. Floor, et al. reported that the gut microbes including Lachnospiraceae, Erysipelotrichaceae, and Clostridiaceae families are involved in protein catabolism ${ }^{[17]}$. Moreover, microbial metabolites including SCFAs increased in rats fed with high protein diets ${ }^{[17]}$.

The nutrients such as protein, fat and carbohydrate can change the community structure and diversity of intestinal microorganisms ${ }^{[10]}$. The nutrient metabolism of nondigestible food components, such as SCFAs, polyamines, polyphenols, and vitamins, produced by intestinal microorganisms, can reprogram the genome through epigenomic mechanisms ${ }^{[18]}$.To sum up, we propose a hypothesis that intestinal microorganisms and microbial metabolites may be related to host nutrient metabolism. The hypothesis may not only provide a new direction for early risk prediction of malnutrition, but also provide novel ideas for the study of nutrition supply in the process of CRC cell proliferation. Clinical investigation shows that serum protein levels in CRC patients are lower than that in the healthy volunteers. To further confirm this hypothesis, we analyzed the gut microbes and microbial metabolites in CRC patients with different serum albumin levels in the present study.

\section{Materials And Methods}

\section{Study design}

The study design is shown in Fig. 1. There are 3 cohorts in this study. Cohort 1, 398 CRC patients at Huzhou Central Hospital from January 2016 to December 2018 were retrospectively analyzed, and 398 age-matched healthy subjects were included from the physical examination center, used to view the differences in serological characteristics between CRC and healthy individuals. Cohort 2, 30 stool samples from CRCs were taken to investigate the gut bacteria and fungus according to different serum 
albumin level. Cohort 3, 56 stool samples from CRCs for the microbial metabolites were investigated according to different serum albumin level.

\section{Subjects}

The patients admitted to the Huzhou Central Hospital and healthy volunteers from the physical examination center in Huzhou Central Hospital were recruited in the study as subjects. The included patients were all Han nationality, all living in Huzhou city, Zhejiang province, People's Republic of China, with similar living habits and eating habits. The clinical trials involving the CRC patients and healthy volunteers and the informed consent form were approved by the Chinese Clinical Trial Registry (http://www.chictr.org.cn, No. ChiCTR1800018908) and Ethics Committee of Huzhou Central Hospital (No.201601022, No.20190204). The inclusion and exclusion criteria of subjects with regard to clinical investigation are shown in Supplemental material 1. All of the stool samples were taken from the above 398 CRC patients in cohort 1. More, patients with known complications such as diabetes, hypertension, smoking, and drinking history over the course of 1 year were excluded in the study of microorganisms and microbial metabolites. The samples used for microbial metabolites study $(n=56)$ covered the samples used for gut microbiota sequencing $(n=30)$. The final included samples for gut microbiota sequencing were those that excluded DNA extraction failure and those that did not meet quality control standards in gut microbiome study.

\section{Clinical data acquisition and stool sample collection}

Basic information and serological indicators with regard to the retrospective study were obtained from the medical record management system of Huzhou Central Hospital and health records system of the physical examination center. The serological indicators were detected by Abbott Automatic Biochemical Analyzer(HCHL-YQ-SH-01). The contents of serum albumin and total protein were determined by bromocresol green method and biuret method, respectively. The results of serological indexes were obtained from the clinical laboratory of Huzhou Central Hospital( Huzhou, Zhejiang province, China). The UICC/AJCC TNM staging system (Version 8, 2017) was used as a standard for clinical staging ${ }^{[19]}$. Approximately $100 \mathrm{~g}$ stool sample was collected and stored in a $-80^{\circ} \mathrm{C}$ freezer within half an hour. The stool samples were analyzed within three months. Freezing the stool samples within half an hour and analyzing within three months was intended to reduce the experimental errors caused by the changes of microorganisms and their metabolites in vitro.

\section{Gut microbe detection}

\subsection{DNA extraction and PCR amplification}


Total DNA from the stool samples was extracted by using the E.Z.N.A. ${ }^{\circledR}$ Stool DNA Kit (Omega Bio-Tek, Norcross, GA, U.S.). DNA integrity was verified using nanodrop ND-1000 spectrophotometer (LabTech, Washington, DC, USA) with the absorbances at $260 \mathrm{~nm}$ and $280 \mathrm{~nm}$ (A260/A280). The quality of the purified DNA was assessed by $2.0 \%(\mathrm{w} / \mathrm{v})$ agarose gel electrophoresis. The V3-V4 region of the bacterial 16S ribosomal DNA gene and fungal ITS ribosomal DNA gene were amplified by PCR. The primers were as follows: bacterial 16S rDNA(630 bp), F 5'-CCTACGGGNGGCWGCAG-3' and R 5'-

GACTACHVGGGTATCTAATCC-3'; ITS rDNA(386 bp): F 5'-CTTGGTCATTTAGAGGAAGTAA-3' and R 5'GCTGCGTTCTTCATCGATGC-3'. The PCR reaction of $25 \mu$ total volume comprised the DNA template $(5 \mu \mathrm{l})$, Nextera XT Index Primer $1(10 \mathrm{M}, 2 \mu \mathrm{l})$, Nextera XT Index Primer $2(10 \mathrm{M}, 2 \mu \mathrm{l})$ and ddH $\mathrm{H}_{2} \mathrm{O}(16 \mu \mathrm{l})$. The conditions for PCR were as follows: $95^{\circ} \mathrm{C}$ for $3 \mathrm{~min}, 25$ cycles at $95^{\circ} \mathrm{C}$ for $30 \mathrm{~s}, 55^{\circ} \mathrm{C}$ for $30 \mathrm{~s}$, and $72^{\circ} \mathrm{C}$ for $45 \mathrm{~s}$ and $72^{\circ} \mathrm{C}$ for $5 \mathrm{~min}$.

\subsection{MiSeq library construction and sequencing}

The amplicons were extracted, purified and quantified by using 2\% agarose gels, AxyPrep DNA Gel Extraction Kit (Axygen Biosciences, Union City, CA, USA) and QuantiFluor ${ }^{\mathrm{TM}}$-ST, respectively. The products were ligated with $Y$ adapter and the self-ligated $Y$ adapters were removed by using magnetic nanoparticles. PCR amplification was conducted for library enrichment and treated with $\mathrm{NaOH}$ solution. The single-stranded DNA denatured by $\mathrm{NaOH}$ were used to construct an Illumina Pair-End library. The Illumina MiSeq platform was used to sequence the amplicon library (Shanghai BIOZERON Co., Ltd.). The raw reads were deposited into the Read Archive (SRA) database of NCBI Sequence (Huzhou Central Hospital : SUB4802613 SRP151510 :PRJNA478277; Huzhou Central Hospital : SUB4648640 SRP169843 : PRJNA506089).

\subsection{Bioinformatics analysis}

The sequencing data were filtered and trimmed, then used for clustering OTUs (operational taxonomic units) and taxonomic analysis. The raw data were optimized as follows: sequences without primers were removed with an allowable error of 0.15 (cutadapt, version 1.11), then PE reads were assembled with an allowable overlap longer than $10 \mathrm{bp}$ (pandaseq, version 2.9) and the ambiguous bases, sequences longer than 300-480 bp, or reads with average quality score less than 20 were discarded. The SILVA database ${ }^{1}$ and the UNITE database were used to analyze and compare the clean reads of 16S rDNA gene sequences and ITS rDNA gene sequences, respectively. The Chao1 indexes, Shannon indexes, and Simpson indexes were used as indicators for the bacterial alpha diversity analysis. Rarefaction curve is a plot of the number of OTUs as a function of the number of sequencing reads. The flattening of the rarefaction curve indicates that the sequencing depth is appropriate. The Shannon and Simpson index indicates the level of microbial diversity of the sample. Higher Shannon and Lower Simpson value indicates higher community diversity. The Mothur software was used to acquire the taxonomy information. RDP-classifier software annotates, Qiime software, and R package HCLUST 
(http://sekhon.berkeley.edu/stats/html/hclust.html) were used to annotate, cluster OTUs, and analyze diversities and community structures.

\section{Microbial metabolites detection 5.1. Sample pretreatment}

After screening and identification, 56 cases of CRC patients were enrolled to perform the gut microbial metabolite detection. Patients included in gut microbial metabolites detection were divided into three groups according to albumin levels. M0, M1, and M2 represent ranges of albumin values of over $40 \mathrm{~g} / \mathrm{L}$, $35 \sim 40 \mathrm{~g} / \mathrm{L}$ and below $35 \mathrm{~g} / \mathrm{L}$, respectively. The samples were pretreated with metabolites extraction, desiccation and derivatization to analyze the microbial metabolites of the stool samples. Stool samples $(10 \mathrm{mg})$ were mixed with $\mathrm{NaOH}$ solution $(300 \mu \mathrm{l})$ and homogenized using a homogenizer (BB24, Next Advance, Inc., Averill Park, NY, USA). The supernatant $(200 \mu \mathrm{l})$ was collected from the mixture after centrifuging at $4^{\circ} \mathrm{C}$ and $16000 \mathrm{rpm}$ for 20 min (Microfuge 20R,Beckman Coulter, Inc., Indianapolis, IN, USA). The residue was homogenized with cold methanol $(200 \mu \mathrm{l})$, centrifuged and $167 \mu \mathrm{L}$ of supernatant was collected. A robotic MultiPurpose Sampler MPS2 with dual heads (Gerstel, Muehlheim, Germany) was used to automatically derive the supernatants. The process was as follows: methyl chloroformate $(20 \mu \mathrm{l})$ was added to the mixture that was shaken for 30 seconds; this step was repeated for the second derivatization; $\mathrm{CHCl}_{3}(400 \mathrm{ml})$ and $\mathrm{Na}_{2} \mathrm{CO}_{3}$ solution $(50 \mathrm{mmol} * 400 \mathrm{ml})$ were added, the mixture was centrifuged at $4^{\circ} \mathrm{C}, 4000 \mathrm{rpm}$ for $20 \mathrm{~min}$; the $\mathrm{CHCl}_{3}$ layer at the bottom was transferred to an auto-sampler vial preloaded with anhydrous $\mathrm{Na}_{2} \mathrm{SO}_{4}(25 \mathrm{mg})$, shaken at $4^{\circ} \mathrm{C}, 1500 \mathrm{rpm}$ for $20 \mathrm{~min}$ and transferred to a capped empty auto-sampler vial for injection.

\subsection{GC/TOFMS analysis}

Each $1 \mu \mathrm{l}$ derivatized sample was injected into an Agilent $6890 \mathrm{~N}$ gas chromatography coupled to time-offlight mass spectrometry (GC-TOFMS) system (Pegasus HT, Leco Corp., St. Joseph, MO, USA). Separation was performed on Rxi-5MS capillary column (Crossbond ® $5 \%$ diphenyl/ $95 \%$ dimethyl polysiloxane) $30 \mathrm{~m}$ (length) x $250 \mu \mathrm{m}$ I.D., $0.25 \mu \mathrm{m}$ film thickness). Helium (99.9999\%) with a flow rate of $1.0 \mathrm{~mL} / \mathrm{min}$ was the carrier gas. The GC temperature gradient was the following: $45^{\circ} \mathrm{C}(1 \mathrm{~min}), 45-260^{\circ} \mathrm{C}\left(20^{\circ} \mathrm{C} / \mathrm{min}\right)$, $260-320^{\circ} \mathrm{C}\left(40^{\circ} \mathrm{C} / \mathrm{min}\right)$ and $320^{\circ} \mathrm{C}(2 \mathrm{~min})$. The electron impact (El) ion was held with a filament bias of $-70 \mathrm{eV}$ at a mass range of $38-550 \mathrm{Da}$ and at $220^{\circ} \mathrm{C}$. The acquisition rate was 20 spectra/second in the MS setting.

\subsection{Differential metabolites identification}

The sample quality control was referred to criterion (IS09001, QAIC/CN/170149, Metabo-Profile, Shanghai, China). The XploreMET software (v2.0, Metabo-Profile, Shanghai, China) was used to process 
the raw data from GC/TOFMS analysis for automatic baseline correction, smoothing, peak picking, peak signal, library searching, and area calculation. The differential metabolites were identified as follows: The GC/MS workstation software was used to determine charge ratio and abundance of characteristic ion fragmentation patterns by comparing the fragment mass automatically. The fragment mass made reference to the available reference standards in the lab (Metabo-Profile Biotechnology Co. Ltd., Shanghai, China), the NISI II standard mass spectral databases, and the Fiehn databases linked to the LECO ChromaTOF software. A similarity of more than $70 \%$ was used as reference standard.

\section{Statistical analysis}

The variables were described with mean \pm standard deviation (SD) or median value and analyzed using the Student's t-test or SNK test, as appropriate. The categorical variables were tested by chi-square test. The SPSS software (version 16.0), Microsoft Excel 2003, and the software packages in RStudio were used as the analysis and mapping software. A two-tailed test result with $p<0.05$ was considered statistically significant. Other statistical methods were noted in the figure or figure legends.

\section{Results}

\section{Serum protein levels in CRCs}

After screening according to the inclusion criteria and exclusion criteria, 398 cases of CRC patients were included in the retrospective study. Age-matched healthy volunteers from the physical examination center were selected as the control group. The clinical characteristics of subjects are shown in Table 1. There was no significant difference between the CRC patients and healthy volunteers in sex, age, BMI, personal habits including smoking and drinking, chronic diseases including diabetes and hypertension, and some serum indicators including hemoglobin, alanine transaminase (ALT), glutamic oxaloacetic transaminase (GOT), creatinine, and triglyceride. Serum nutrition-related indicators including total protein and albumin in CRC group were lower than those in control group ( $\mathrm{p} \otimes 0.05)$. Other serum biochemical indicators such as high density lipoprotein (HDL), low density lipoprotein ( $L D L)$, and Apolipoprotein $A(a p o A)$ were also statistically different between these two groups. 
Table 1

Characteristics of the Subjects with Regard to Clinical Investigation

\begin{tabular}{|c|c|c|c|}
\hline & Colorectal Cancer group & Healthy Control group & $\mathrm{p}$ value \\
\hline Cases, $\mathrm{n}$ & 398 & 398 & / \\
\hline Males, n & 236 & 231 & 0.669 \\
\hline Age, years & $64.59 \pm 9.13$ & $64.21 \pm 8.89$ & 0.555 \\
\hline $\mathrm{BMI}, \mathrm{kg} / \mathrm{m}^{2}$ & $22.39 \pm 3.02$ & $22.45 \pm 2.84$ & 0.793 \\
\hline Long-term smoking history, $\mathrm{n}$ & 98 & 88 & 0.373 \\
\hline Long-term drinking history, $\mathrm{n}$ & 64 & 63 & 0.920 \\
\hline Known diabetes, $\mathrm{n}$ & 26 & 20 & 0.448 \\
\hline Known hypertension, $\mathrm{n}$ & 98 & 88 & 0.451 \\
\hline Hemoglobin (g/L) & $122.12 \pm 21.15$ & $124.71 \pm 20.93$ & 0.082 \\
\hline Alanine transaminase (U/L) & $17.09 \pm 9.93$ & $16.85 \pm 9.72$ & 0.731 \\
\hline $\begin{array}{l}\text { Glutamic oxaloacetic transaminase } \\
(\mathrm{U} / \mathrm{L})\end{array}$ & $21.56 \pm 6.50$ & $21.62 \pm 7.23$ & 0.890 \\
\hline Total protein (g/L) & $65.03 \pm 6.58$ & $70.73 \pm 8.32$ & 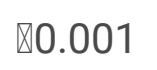 \\
\hline Albumin $(g / L)$ & $39.25 \pm 4.29$ & $44.97 \pm 3.34$ & $\varangle 0.001$ \\
\hline Globulin (g/L) & $25.78 \pm 4.19$ & $25.75 \pm 7.42$ & 0.952 \\
\hline Creatinine $(\mu \mathrm{mol} / \mathrm{L})$ & $68.95 \pm 16.60$ & $68.86 \pm 16.73$ & 0.941 \\
\hline Triglyceride (mmol/L) & $1.35 \pm 0.66$ & $1.26 \pm 0.59$ & 0.054 \\
\hline $\mathrm{HDL}(\mathrm{mg} / \mathrm{dL})$ & $44.20 \pm 12.24$ & $96.29 \pm 26.07$ & 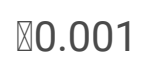 \\
\hline LDL (mg/dL) & $100.41 \pm 27.11$ & $20.41 \pm 15.89$ & $\otimes 0.001$ \\
\hline Apol a (mg/dL) & $28.56 \pm 28.22$ & $20.42 \pm 15.89$ & $\otimes 0.001$ \\
\hline \multicolumn{4}{|c|}{$\begin{array}{l}\text { The clinical stages conformed to the criterion of CRC according to the UICC/AJCC TNM staging } \\
\text { system (Version } 8,2017) \text {. Smoking and drinking history over the course of } 1 \text { year were collected. } \\
\text { Serum nutrition-related indicators from CRC patients before treatments including chemotherapy, } \\
\text { radiation, and surgery were collected. Chi-square test and student t-test was used for counting data } \\
\text { comparison and measuring data comparison, respectively. }\end{array}$} \\
\hline
\end{tabular}

Clinically, serum albumin level is an objective index for nutritional assessment. HDL, LDL and Apo A are not used as an indicator of nutritional assessment. We further analyzed the nutrition-related indicators including total protein, albumin, and globulin, and the ratio of albumin and globulin in CRC patients in terms of gender, pathological stage, and cancer site. As shown in Table 2, there was no significant 
difference in the serum nutrition-related indicators at different pathological stages and between males and females. There was no significant difference in the globulin levels and in the ratio of albumin and globulin between the colon cancers and rectal cancers, but the total protein and albumin in colon cancer was lower than that in rectal cancer $(p<0.05)$.

\section{Association of gut microbes with different serum albumin levels in CRCs}


Table 2

Serum nutrition-related indicators in CRCs with respect to gender, pathological stages, and cancer sites

\begin{tabular}{|c|c|c|c|c|c|c|}
\hline Groups & Subgroups & Cases & Total protein & Albumin & Globulin & Albumin/ Globulin \\
\hline $\begin{array}{l}\text { Reference } \\
\text { ranges }\end{array}$ & & - & $65-85 \mathrm{~g} / \mathrm{L}$ & $\begin{array}{l}40- \\
55 \mathrm{~g} / \mathrm{L}\end{array}$ & $\begin{array}{l}20- \\
40 \mathrm{~g} / \mathrm{L}\end{array}$ & $1.20-2.40$ \\
\hline $\begin{array}{l}\text { Colorectal } \\
\text { cancer }\end{array}$ & & 398 & $65.03 \pm 6.58$ & $\begin{array}{l}39.25 \pm \\
4.29\end{array}$ & $\begin{array}{l}25.78 \pm \\
4.19\end{array}$ & $1.56 \pm 0.28$ \\
\hline \multirow[t]{5}{*}{$\begin{array}{l}\text { Pathological } \\
\text { stages }\end{array}$} & Stage I & 64 & $63.45 \pm 5.01$ & $\begin{array}{l}38.67 \pm \\
3.85\end{array}$ & $\begin{array}{l}24.79 \pm \\
3.69\end{array}$ & $1.60 \pm 0.31$ \\
\hline & Stage II & 270 & $64.87 \pm 6.67$ & $\begin{array}{l}39.24 \pm \\
4.43\end{array}$ & $\begin{array}{l}25.63 \pm \\
3.95\end{array}$ & $1.56 \pm 0.26$ \\
\hline & Stage III & 64 & $67.25 \pm 7.05$ & $\begin{array}{l}39.84 \pm \\
4.08\end{array}$ & $\begin{array}{l}27.41 \pm \\
5.17\end{array}$ & $1.50 \pm 0.31$ \\
\hline & $\mathrm{F}$ value & - & 5.697 & 1.194 & 6.987 & 1.910 \\
\hline & $\mathrm{p}$ value & - & 0.153 & 0.175 & 0.186 & 0.068 \\
\hline \multirow[t]{4}{*}{ Sex } & Male & 236 & $64.61 \pm 6.67$ & $\begin{array}{l}38.96 \pm \\
4.28\end{array}$ & $\begin{array}{l}25.65 \pm \\
4.05\end{array}$ & $1.55 \pm 0.26$ \\
\hline & Female & 162 & $65.63 \pm 6.41$ & $\begin{array}{l}39.66 \pm \\
4.28\end{array}$ & $\begin{array}{l}25.97 \pm \\
4.39\end{array}$ & $1.57 \pm 0.30$ \\
\hline & $\mathrm{t}$ value & - & 1.519 & 1.587 & 0.759 & 0.664 \\
\hline & $p$ value & - & 0.130 & 0.113 & 0.448 & 0.507 \\
\hline \multirow[t]{4}{*}{ Sites } & Colon & 188 & $64.34 \pm 6.47$ & $\begin{array}{l}38.77 \pm \\
4.39\end{array}$ & $\begin{array}{l}25.57 \pm \\
3.93\end{array}$ & $1.55 \pm 0.27$ \\
\hline & Rectum & 210 & $65.64 \pm 6.63$ & $\begin{array}{l}39.67 \pm \\
4.17\end{array}$ & $\begin{array}{l}25.96 \pm \\
4.42\end{array}$ & $1.57 \pm 0.28$ \\
\hline & $\mathrm{t}$ value & - & 1.978 & 2.118 & 0.940 & 0.678 \\
\hline & $p$ value & - & 0.049 & 0.035 & 0.348 & 0.498 \\
\hline \multicolumn{7}{|c|}{$\begin{array}{l}\text { The serum nutrition-related indicators in CRCs with respect to gender, pathological stages, and cancer } \\
\text { sites were investigated and analyzed. The clinical stages conformed to the criterion of CRC according } \\
\text { to the UICC/AJCC TNM staging system (Version 8, 2017). Serum nutrition-related indicators from CRC } \\
\text { patients before treatments including chemotherapy, radiation, and surgery were collected. } \\
\text { Independent sample t- test was used for comparison of measurement data between the two groups, } \\
\text { and SNK test was used among the three groups. }\end{array}$} \\
\hline
\end{tabular}

A total of 30 cases of CRC patients were included to detect the gut microbiota. These patients were divided into three groups according to albumin levels. F0, F1, and F2 represent ranges of albumin values of over $40 \mathrm{~g} / \mathrm{L}, 35 \sim 40 \mathrm{~g} / \mathrm{L}$ and below $35 \mathrm{~g} / \mathrm{L}$, respectively. As shown in Table 3, there was no significant 
difference among these three groups in sex, age, BMI, and some serum indicators including hemoglobin, ALT, GOT, creatinine, triglyceride, HDL, LDL, and apolA.

Table 3

Characteristics of the study participants with regard to microbiota investigation

\begin{tabular}{|c|c|c|c|c|}
\hline & Fo & F1 & F2 & $p$ value \\
\hline Cases, n & 8 & 14 & 8 & / \\
\hline Males, n & 4 & 7 & 6 & 0.474 \\
\hline Age, years & $64.13 \pm 9.73$ & $64.71 \pm 6.58$ & $65.00 \pm 8.45$ & 0.975 \\
\hline $\mathrm{BMI}, \mathrm{kg} / \mathrm{m}^{2}$ & $21.52 \pm 2.34$ & $21.78 \pm 3.60$ & $22.60 \pm 1.83$ & 0.736 \\
\hline Hemoglobin(g/L) & $120.88 \pm 15.64$ & $115.07 \pm 20.24$ & $109.13 \pm 25.85$ & 0.537 \\
\hline $\operatorname{ALT}(\mathrm{U} / \mathrm{L})$ & $24.89 \pm 23.94$ & $14.00 \pm 6.12$ & $15.95 \pm 6.96$ & 0.193 \\
\hline GOT(U/L) & $22.61 \pm 11.77$ & $19.69 \pm 4.78$ & $19.65 \pm 5.31$ & 0.632 \\
\hline Total protein(g/L) & $70.81 \pm 4.23$ & $62.26 \pm 3.26 *$ & $55.34 \pm 5.22^{\#}$ & $\bigotimes 0.001$ \\
\hline Albumin(g/L) & $43.98 \pm 1.67$ & $37.61 \pm 1.50^{\star}$ & $33.11 \pm 1.40^{\#}$ & 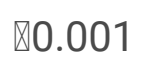 \\
\hline Globulin(g/L) & $26.84 \pm 2.65$ & $24.64 \pm 2.90$ & $22.21 \pm 4.46$ & 0.033 \\
\hline Creatinine $(\mu \mathrm{mol} / \mathrm{L})$ & $62.90 \pm 8.12$ & $63.30 \pm 12.64$ & $80.01 \pm 32.50$ & 0.123 \\
\hline Triglyceride(mmol/L) & $1.11 \pm 0.31$ & $1.24 \pm 0.61$ & $1.19 \pm 0.60$ & 0.859 \\
\hline HDL (mg/dL) & $52.25 \pm 10.39$ & $46.14 \pm 15.85$ & $39.95 \pm 10.24$ & 0.198 \\
\hline LDL (mg/dL) & $84.72 \pm 35.99$ & $99.55 \pm 25.95$ & $83.90 \pm 17.10$ & 0.323 \\
\hline ApolA(mg/dL) & $37.39 \pm 28.20$ & $27.97 \pm 22.63$ & $33.80 \pm 23.06$ & 0.667 \\
\hline \multicolumn{5}{|c|}{$\begin{array}{l}\text { F0, F1, and F2 represent ranges of albumin values of over } 40 \mathrm{~g} / \mathrm{L}, 35 \sim 40 \mathrm{~g} / \mathrm{L} \text { and below } 35 \mathrm{~g} / \mathrm{L} \text {, } \\
\text { respectively. Serum nutrition-related indicators from CRC patients before treatments including } \\
\text { chemotherapy, radiation, and surgery were collected. Patients with known complications such as } \\
\text { diabetes, hypertension, smoking, and drinking history over the course of } 1 \text { year were excluded. Three } \\
\text { groups of measurement data were compared using SNK test.(* means that } p<0.05 \text {, compared to the } \\
\text { F0 group, }{ }^{*} \text { means that } p<0.05 \text {, compared to the F1 group.) }\end{array}$} \\
\hline
\end{tabular}

The gut bacteria were detected to compare the diversity among the three groups. As shown in Supplement material-Figure S1, panels A, B, and C show the Chao1 curves, Shannon curves, and Simpson curves, respectively. There is no statistical difference (Kruskal test) between Chao1 indexes, Shannon indexes, and Simpson indexes among the three groups.

The community structure of gut bacteria among the three groups was analyzed. Figure 2-4 shows the plot of community structure among the three groups. The stacked bar graph in Fig. 2 shows the relative 
abundance of gut bacteria at the genus level for each sample. Despite the large variation of the bacteria in each sample, the bacteria that made up the majority of stool samples in CRC patients, in turn, included Bacteroides, Escherichia/Shigella, Prevotella, Ruminococcaceae, Faecalibacterium, Streptococcus, Roseburia, Parabacteroides, Lachnospiraceae, and Clostridium XIVa. The taxonomic tree heatmap in Fig. 3 shows the composition and proportion of microorganisms at different taxonomic levels including phylum, class, order, family, and genus. The outermost layer indicates annotated genus. The orange circles indicate that the abundance of Sutterella (phylum_Proteobacteria, class_Betaproteobacteria, order_Burkholderiales, family_Sutterellaceae, genus_sutterella) is higher in the CRCs with the lower serum albumin level (Kruskal test). The enterotype map in Fig. 4 represents the correlation and contribution of the bacteria to different groups. Orange area, cyan area, and purple area represents F0 group, F1 group, and F2 group, respectively. The result shows that Burkholderia, Grenulicatella, Stenotrophomonas, Collinsella, Actinomyces, Oribacterium, Delftia, and Parasutterella are closely related to F0 group. Ruminococcus, Coprococcus, and Paraprevotella are closely related to F1 group. Prevotella, f_Porphyromonadaceae, Alloprevotella, Methanobrevibacter, Porphyromonas, Coprobacter, Eggerthia, Lactobacillus, Turicibacter, Pseudoflavonifractor, Campylobacter, Sutterella, and Oscilfibacter are closely related to F2 group (Monte-Carlo simulation).

The gut fungus were detected to compare the diversity among the three groups. The Supplement material-Figure S2 shows the alpha diversity analysis of gut fungus among the three groups including F0, F1, and F2. Panels A, B, and C shows the Chao1 curves, Shannon curves, and Simpson curves, respectively. There is no statistical difference (Kruskal test) between Chao1 indexes, Shannon indexes, and Simpson indexes among the three groups.

Figure 5-7 shows the community structure of gut fungus among the three groups. The stacked bar graph in Fig. 5 shows the relative abundance of gut fungus from the stool samples of CRC patients at the genus level. The fungus that can be successfully compared by the relative database in the top 10 include Saccharomyces, Saccharomycetales, Humicola, Candida, Rhodotorula, Ascomycota, Penicillium, Malassezia, Filobasidium, and Debaryomyces. The taxonomic tree heatmap in Fig. 6 shows that there were statistically significant differences in the abundance of fungus including Agaricomycetes (p_Basidiomycota, c_Agaricomycetes, o_Agaricomycetes_unidentified,

f_Agaricomycetes_unidentified_1, g_Agaricomycetes), Simplicillium (p_Ascomycota, c_Sordariomycetes, o__Hypocreales, f_Cordycipitaceae, g_Simplicillium), Sclerotiniaceae

(p_Ascomycota, c_Leotiomycetes, o_Helotiales, f_Sclerotiniaceae, g_Sclerotiniaceae), and Exophiala (p_Ascomycota, c_Eurotiomycetes, o_Chaetothyriales, f_Herpotrichiellaceae, g_Exophiala) among the three groups (Kruskal test). The enterotype map in Fig. 7 represents the correlation and contribution of the gut fungus to different groups. Orange area, cyan area and purple area represent F0 group, F1 group, and F2 group, respectively. The result shows that Simplicillium, Bionectria, Verticillium, Hannaella, Davidiella, Thanatephorus, Acrostalagmus, Devriesia, Malassezia, Cyberlindnera, Trichocomaceae, and Asterotremella are closely related to F0group. Eurotiomycetes and Aspergillus are closely related to F1 group. Bionectraceae, Magnusiomyces, Sarocladium, Phoma. Kodamaea, Pichia, Pseudogymnoascus, 
Incertae_sedis, Trichosporon, Geotrichum, Penicillium, Auricularia, and Pleosporaceae are closely related to F2 group (Monte-Carlo simulation).

\section{Association of gut microbial metabolites with different serum albumin levels in CRCs}

As shown in Table 4, there was no significant difference among these three groups with respect to sex, age, BMI, and some serum indicators including hemoglobin, ALT, GOT, creatinine, triglyceride, HDL, LDL, and apolA. A total of 124 microbial metabolites in 10 classes extracted from stool samples of CRC patients were quantified in the present study. The list of microbial metabolites is showed in Supplement material-table S1. 
Table 4

Characteristics of the study participants with regard to microbial metabolites investigation

\begin{tabular}{|c|c|c|c|c|}
\hline & Mo & M1 & M2 & $p$ value \\
\hline Cases, n & 14 & 28 & 14 & \\
\hline Males, n & 5 & 14 & 9 & 0.215 \\
\hline Age, years & $63.52 \pm 9.00$ & $64.95 \pm 6.59$ & $66.53 \pm 6.69$ & 0.442 \\
\hline $\mathrm{BMI}, \mathrm{kg} / \mathrm{m}^{2}$ & $21.87 \pm 2.60$ & $22.21 \pm 3.64$ & $21.70 \pm 2.69$ & 0.823 \\
\hline Hemoglobin $(\mathrm{g} / \mathrm{L})$ & $122.26 \pm 12.58$ & $118.43 \pm 18.88$ & $109.47 \pm 21.11$ & 0.069 \\
\hline Alanine transaminase $(\mathrm{U} / \mathrm{L})$ & $19.77 \pm 11.61$ & $14.89 \pm 5.37$ & $17.70 \pm 7.78$ & 0.161 \\
\hline $\begin{array}{l}\text { Glutamic oxaloacetic transaminase } \\
(\mathrm{U} / \mathrm{L})\end{array}$ & $22.87 \pm 9.99$ & $20.70 \pm 4.38$ & $22.39 \pm 6.47$ & 0.412 \\
\hline Total protein (g/L) & $71.07 \pm 4.22$ & $62.34 \pm 3.56^{\star}$ & $57.16 \pm 5.95^{\#}$ & $\llbracket 0.001$ \\
\hline Albumin $(g / L)$ & $43.67 \pm 2.10$ & $37.76 \pm 1.52^{\star}$ & $32.87 \pm 1.35^{\#}$ & $\llbracket 0.001$ \\
\hline Globulin (g/L) & $27.39 \pm 3.19$ & $24.57 \pm 3.10$ & $24.28 \pm 5.28$ & 0.008 \\
\hline Creatinine $(\mu \mathrm{mol} / \mathrm{L})$ & $64.64 \pm 8.31$ & $65.02 \pm 17.82$ & $67.26 \pm 21.90$ & 0.904 \\
\hline Triglyceride (mmol/L) & $1.26 \pm 0.59$ & $1.26 \pm 0.57$ & $1.00 \pm 0.45$ & 0.210 \\
\hline $\mathrm{HDL}(\mathrm{mg} / \mathrm{dL})$ & $49.14 \pm 10.07$ & $45.94 \pm 14.54$ & $42.50 \pm 9.47$ & 0.232 \\
\hline LDL (mg/dL) & $106.43 \pm 31.61$ & $10005 \pm 24.51$ & $102.14 \pm 31.79$ & 0.789 \\
\hline Apolipoprotein a (mg/dL) & $31.52 \pm 26.45$ & $30.64 \pm 34.28$ & $37.78 \pm 24.90$ & 0.686 \\
\hline \multicolumn{5}{|c|}{$\begin{array}{l}\text { M0, M1 and M2 represent ranges of albumin values of over } 40 \mathrm{~g} / \mathrm{L}, 35 \sim 40 \mathrm{~g} / \mathrm{L} \text { and below } 35 \mathrm{~g} / \mathrm{L} \text {, } \\
\text { respectively. Serum nutrition-related indicators from CRC patients before treatments including } \\
\text { chemotherapy, radiation, and surgery were collected. Patients with known complications such as } \\
\text { diabetes, hypertension, smoking, and drinking history over the course of } 1 \text { year were excluded. Three } \\
\text { groups of measurement data were compared using SNK test. (* means that } \mathrm{p}<0.05 \text {, compared to the } \\
\text { M0 group, " means that } p<0.05 \text {, compared to the M1 group.) }\end{array}$} \\
\hline
\end{tabular}

Figure 8 shows the relative proportion of gut microbial metabolites in each example. The stacked bar on the left represents each sample, and the stacked bar on the right represents a set of the same group. Panel A in Fig. 8 shows the proportion of gut microbial metabolites in the detected samples at class level. The results show that the gut microbial metabolites accounting for the top three include amino acids, cinmic acids, and fatty acids at class level. Panel B in Fig. 8 shows the proportion of gut microbial metabolites in the absolute quantitative top 30. The gut microbial metabolites from CRC patients in the top 10 including acetic acid, L-methionine, 3-hydroxybutyric acid, L-glutamic acid, L-lysine, glutathione, glyceric acid, oxoadipic acid, L-homoserine, and gamma-aminobutyric acid. The heatmap in Fig. 9 shows that the gut microbial metabolites had statistically significant differences among the three groups 
(Kruskal test). The panel A and panel B in Fig. 9 shows the concentration of gut microbial metabolites from each sample and each group, respectively. The results show that the stool samples in CRC patients with the lower serum albumin level had an increase in the concentration of gut microbial metabolites including 2-hydroxybutyric acid, 2-phenylglycine, D-2-hydroxyglutaric acid, glycine, L-asparagine, behenic acid, oxoglutaric acid and succinic acid, and a decrease in the concentration of gut microbial metabolites including L-norleucine, salicyluric acid, isocitric acid, and glycine.

\section{Discussion}

In the present study, serum albumin levels in CRC patients were retrospectively analyzed and compared with those of healthy controls. We found that serum albumin levels decreased in CRC patients. The total protein and albumin in colon cancer was lower than that in rectal cancer. The abundance of Sutterella is higher in the CRCs with the lower serum albumin level. There were statistically significant differences of some gut fungus including Agaricomycetes, Simplicillium, Sclerotiniaceae, and Exophiala in different serum albumin levels. The concentration of gut microbial metabolites including 2-hydroxybutyric acid, 2phenylglycine, D-2-hydroxyglutaric acid, glycine, L-asparagine, behenic acid, oxoglutaric acid and succinic acid were increased in CRC patients with the lower serum albumin level. And some microbial metabolites including L-norleucine, salicyluric acid, isocitric acid, and glycine were decreased.

Some factors are considered to cause errors in the detection results of intestinal microorganisms. Therefore, patients with known complications such as diabetes, hypertension, smoking, and drinking history over the course of 1 year were excluded. In addition, excluded DNA extraction failure and those that did not meet quality control standards in the process of measuring gut microbiome. Finally, 30 cases were included in the study of microorganisms and 56 cases were including in microbial metabolites. The CRCs patients were divided into three groups according to albumin levels, including $\mathrm{FO}$ (or $\mathrm{M0}$ ) for over $40 \mathrm{~g} / \mathrm{L}, \mathrm{F} 1$ (or M1) for 35-40 g/L, and F2(or M2) for below $35 \mathrm{~g} / \mathrm{L}$. According to the reference value range of clinical serological indicators in the clinical laboratory department of the hospital, serum albumin less than $40 \mathrm{~g} / \mathrm{L}$ was considered as hypoproteinemia. The guidelines for nutritional therapy for cancer patients are not clearly defined. Serum albumin less than $35 \mathrm{~g} / \mathrm{L}$ was used as the reference standard for

hypoproteinemia in some studies of colorectal cancer patients with hypoproteinemia ${ }^{[20,21]}$. Taking into account the retrospective analysis of serum albumin levels in CRC and the balance of the number of patients included in the group. Therefore, this grouping criteria were selected.

Most previous studies have focused on the effects of high-protein diets on gut microbes ${ }^{[13,22]}$. Protein ingested by the human body is first broken down into amino acids, converted into other metabolites by transamination or excreted by deamination and oxidative decomposition, a process known as nitrogen balance $^{[23-25]}$. Nitrogen balance determines that a high-protein diet does not change the body's serum protein and albumin level. Thus, whether a high-protein diet has a positive or negative effect on the body is debatable. However, the negative nitrogen balance caused by cancers, especially the malignant tumors in the digestive tract, leads to malnutrition, having a negative impact on health ${ }^{[26]}$. Serum total protein mainly includes globulin and albumin, and the level of total protein and albumin reflects the nutritional 
status of patients. Therefore, studying the relationship between negative nitrogen balance and gut microbes is essential.

The present study found that serum albumin levels decreased in CRC patients. This predictable result has been confirmed by other studies ${ }^{[27,28]}$. However, we also found that the total protein and albumin in colon cancer was lower than that in rectal cancer. Besides the difference in the anatomical site, there could be other factors, such as bleeding, intestinal obstruction and tumor growth rate, contributing to hypoproteinemia. Factors leading to hypoproteinemia and effect of hypoproteinemia on the prognosis of $\mathrm{CRC}$ need further investigation.

We found that the top three bacteria in the stool sample from CRCs were Bacteroides, Escherichia/Shigella, and Prevotella. The top three bacteria association with CRC has also been previously reported. For instance, several studies suggested that Bacteroides are the bacteria that induce $\mathrm{CRC}^{[29,30]}$. Tingting, et al. reported that Escherichia/Shigella were present at higher concentrations in the stool of CRC patients that in stools of healthy volunteers ${ }^{[31]}$. A review showed that gut Prevotella was correlated with diets and non-communicable diseases including $\mathrm{CRC}^{[32]}$.

The results in the present study showed that the higher abundance of Sutterella is correlated with lower serum albumin level in CRCs. Sutterella is a commensal that can adhere to intestinal epithelial cells and promote inflammation and has been associated with human diseases, such as inflammatory bowel disease (IBD) ${ }^{[33]}$. IBD induces CRC through repeated cycles of epithelial injury and repair ${ }^{[34]}$. However, the relationship between hypoproteinemia and Sutterella in CRC remains unclear. The correlation between nutritional status and the species level and subspecies level with regard to the genus Sutterella is worth further study.

In recent years, more attention has been paid to the study of gut fungus ${ }^{[35]}$. Our results show that the fungus Saccharomyces, Saccharomycetales, and Humicola accounted for the top three in stool samples of CRC patients. Glinka, et al. reported a result consistent with our study that the abundance of Saccharomyces genus was increased in gastrointestinal malignancies ${ }^{[36]}$. Heat-killed form of Saccharomyces cerevisiae acted as a probiotic and caused apoptosis in colon cancer cells ${ }^{[37]}$. Such reports with regard to Humicola in cancer are rare. Harsimran, et al. reported that they isolated Ochroconis humicola from the tissue of esthesioneuroblastoma ${ }^{[38]}$, but not CRC. There was rarely reported on the role in nutrition status. fungus, as an important component of microorganisms, should not be overlooked in CRC research. We're still in the infancy stage of studying the complex relationship among fungus, CRC, and nutrition.

There are multiple studies on the microbial metabolites short chain fatty acids (SCFAs) in relation to CRC and nutrition ${ }^{[9,39-41]}$. The metabolomics method including 124 microbial metabolites allows for a more comprehensive assessment of stool samples from CRC patients. The results showed that some microbial metabolites, such as acetic acid, L-methionine, 3-hydroxybutyric acid, L-glutamic acid, and L-lysine, were relatively higher in the fecal samples from CRC patients than healthy subjects. It has been studied 
extensively that acetic acid, as one of the components of SCFAs, functions in the suppression of CRC [42]. The recombinant L-methionine gamma-lyase from Brevibacterium aurantiacum can eliminate Lmethionine and induce growth arrest and death of $\mathrm{CRC}$ cells ${ }^{[43]}$. Yui, et al. reported that 3-hydroxybutyric acid can reprogram energy metabolism during glucose starvation and inhibit the proliferation of CRC cells ${ }^{[44]}$. The supplementation of glutamic acid can decrease the risk of $\mathrm{CRCs}^{[45,46]}$. We found some characteristic gut microbial metabolites to be increased or decreased in CRC patients correlating with the lower serum albumin level in the present study. Due to the lack of similar reports and the insufficient sample size in this study, the results need to be verified by multi-center experiments with larger sample size.

It is of great significance to study the association between nutritional status and gut microbes and microbial metabolites in CRC. Firstly, it may provide a novel direction to translational medicine in the assessment of nutritional status from the perspective of microbiology. Secondly, stool samples have the advantages of no innovation and easy access. The correlation analysis of gut bacteria and fungus with different albumin levels may provide some basis for building a network of interactions between microbes and nutrients. Specifically, the network contains the relationship between the microbes including diversity and community structure of bacteria and fungus, quantification of microbial metabolites and nutrients including proteins, fats, and carbohydrates. The dynamic monitoring of characteristic microbes and microbial metabolites in the stool may provide a simple and rapid early warning and screening method for malnutrition. Thirdly, some means of intervention, such as fecal transplantation, oral administration of microorganisms, supplementation of microbial metabolites, and dietary choices may provide alternative treatments and auxiliary treatment for patients with malnutrition.

We acknowledge the shortcomings of this study. Considering that the investigation may be influenced by a variety of factors such as geographical location, nosocomial infection, or pollution contamination in the fecal collection process, we enrolled CRC patients from the same region and ethnic group to minimize the influence of diet on intestinal microorganisms. More, the strict detection standards, sample collection standards, inclusion and exclusion criteria were conducted in the present study. However, the influence of individual dietary habits and life style on microecology is inevitable. In addition, many ITS sequences cannot be annotated. The 125 microbial metabolites are not the full spectrum of microbial metabolites. The investigation needs to be further in-depth and verified.

The interrelation and interactions between microbes and metabolites in the intestinal tract are very complex. There are obvious defects in using single microbes or metabolites to predict the nutritional status. Our future plans include acquiring larger data of microbiology and microbial metabolomics by including polycentric, multi-regional samples. A more complete and scientific nutritional status assessment model will be developed by applying machine learning models, such as neural network model or random forest model. These larger studies should provide clinical guidance for nutritional assessment and intervention.

\section{Conclusions}


The serum total protein and serum albumin levels in CRC group were lower than those in healthy people. We determined characteristic gut microbes and microbial metabolites that correlated with the different serum albumin levels in CRCs, and our findings further support the hypothesis that intestinal microorganisms and microbial metabolites may be related to host nutrient metabolism. It may provide novel ideas for basic research and clinical application.

\section{List Of Abbreviations}




\begin{tabular}{|ll|}
\hline Abbreviation & Definition \\
\hline CRC & Colorectal Cancer \\
\hline GS & Gas Chromatography \\
MS & Mass Spectrometer \\
OTU & Multidisciplinary comprehensive treatment \\
\hline SD & Operational taxonomic unit \\
AJCC & Standard deviation \\
\hline NCCN & American Joint Committee on Cancer \\
UICC & National Comprehensive Cancer Network \\
PCR & Polymerase Chain Reaction \\
\hline rDNA & ribosomal Deoxyribonucleic Acid \\
\hline MCF & Methyl chloroformate \\
\hline TOFMS & Time-of-Flight Mass Spectrometry \\
\hline EI & Electron lonization \\
\hline FBG & Fasting Blood Glucose \\
\hline OTU & Operational Taxonomic Unit \\
ALT & Alanine transaminase \\
GOT & Glutamic oxaloacetic transaminase \\
\hline HDL & High density lipoprotein \\
\hline LDL & Low density lipoprotein \\
\hline apoA & Apolipoprotein a \\
\hline BFT & Bacteroides fragilis toxin \\
\hline IBD & \\
\hline
\end{tabular}

\section{Declarations}

\section{Ethics approval and consent to participate}

The clinical protocols involving the patients and the informed consent form were approved by the Chinese Clinical Trial Registry (http://www.chictr.org.cn, No. ChiCTR1800018908) and Ethics Committee 
of Huzhou Central Hospital (No. 201601022, No. 20190204).

Consent for publication

Not applicable.

\section{Availability of data and materials}

The datasets generated during the current study are not publicly available but obtained from corresponding authors on reasonable request.

\section{Competing interests}

The authors declare that no conflicts of interest exist.

\section{Funding}

This work was supported by the Major Science and Technology Projects for Medical and Health Care of Zhejiang Province(No. WKJZJ-2013), Zhejiang Medical and Health Technology Projects (No. 2019RC283) and Public Welfare Technology Application Research Program of Huzhou (No. 2018GY15).

\section{Authors' contributions}

All authors participated in the conception and design of the study;

Conceived and drafted the manuscript: Han Shuwen and Yang Xi;

Performed the experiments: Wu Wei, Da Miao ;

Collected the basic patient information, clinical indicators, and imaging data: Zhuang Jing and Xu Jiamin;

Analyzed the data: Zhou Qing and Liu Jin ;

Wrote the paper: Han Shuwen and Yang Xi;

All authors read and approved the paper.

\section{Acknowledgements}

We thank the patients and volunteers for their contributions to the investigation of clinical data, serological indexes, and sample collection.

\section{References}


1. Siegel RL, Miller KD, Fedewa SA, et al. Colorectal cancer statistics, 2017. CA Cancer J Clin. 2017;67(3):177-193.

2. Lin WL, Sun JL, Chang SC, et al. Effectiveness of the Multidisciplinary Team Model in Treating Colorectal Cancer. Gastroenterol Nurs. 2018;41(6):491-496.

3. Fan JQ, Shang D, Han B, et al. Adoptive Cell Transfer: Is it a Promising Immunotherapy for Colorectal Cancer? Theranostics. 2018;8(20):5784-5800.

4. Luo DK, Liu Q, Zhu J, et al. Survival Benefit of Preoperative Versus Postoperative Radiotherapy in Metastatic Rectal Cancer Treated With Definitive Surgical Resection of Primary Tumor: A Population Based, Propensity Score-Matched Study. Journal of Cancer. 2019;10(5):1307-1312.

5. Mima K, Ogino S, Nakagawa S, et al. The role of intestinal bacteria in the development and progression of gastrointestinal tract neoplasms. Surg Oncol. 2017;26(4):368-376.

6. Liang Q, Chiu J, Chen Y, et al. Fecal Bacteria Act as Novel Biomarkers for Noninvasive Diagnosis of Colorectal Cancer. Clin Cancer Res. 2017;23(8):2061-2070.

7. Chen J, Pitmon E, Wang K. Microbiome, inflammation and colorectal cancer. Semin Immunol. 2017;32:43-53.

8. Wang S, Dong W, Liu L, et al. Interplay between bile acids and the gut microbiota promotes intestinal carcinogenesis. Mol Carcinog. 2019.

9. Wang G, Yu Y, Wang YZ, et al. Role of SCFAs in gut microbiome and glycolysis for colorectal cancer therapy. J Cell Physiol. 2019.

10. Han S, Gao J, Zhou Q, et al. Role of intestinal flora in colorectal cancer from the metabolite perspective: a systematic review. Cancer Manag Res. 2018;10:199-206.

11. Han S, Pan $Y$, Yang $X$, et al. Intestinal microorganisms involved in colorectal cancer complicated with dyslipidosis. Cancer Biol Ther. 2019;20(1):81-89.

12. Xu J, Verbrugghe A, Lourenco $M$, et al. The response of canine faecal microbiota to increased dietary protein is influenced by body condition. Bmc Veterinary Research. 2017;13.

13. Liu JP, Zou WL, Chen SJ, et al. Effects of different diets on intestinal microbiota and nonalcoholic fatty liver disease development. World J Gastroenterol. 2016;22(32):7353-7364.

14. Mu C, Yang Y, Luo Z, et al. Temporal microbiota changes of high-protein diet intake in a rat model. Anaerobe. 2017;47:218-225.

15. He X, Sun W, Ge T, et al. An increase in corn resistant starch decreases protein fermentation and modulates gut microbiota during in vitro cultivation of pig large intestinal inocula. Anim Nutr. 2017;3(3):219-224.

16. Pinna C, Vecchiato CG, Bolduan C, et al. Influence of dietary protein and fructooligosaccharides on fecal fermentative end-products, fecal bacterial populations and apparent total tract digestibility in dogs. BMC Vet Res. 2018;14(1):106.

17. Floor $H$, Mark D , Jessica $S$, et al. Metatranscriptome analysis of the microbial fermentation of dietary milk proteins in the murine gut[J]. PLOS ONE, 2018, 13(4):e0194066-. 
18. Bhat M I , Kapila R. Dietary metabolites derived from gut microbiota: critical modulators of epigenetic changes in mammals[J]. Nutrition Reviews, 2017.

19. Lynch HT, Shaw TG. Practical genetics of colorectal cancer. Chin Clin Oncol. 2013;2(2):12.

20. Egenvall M , M. Mörner, Martling A, et al. Prediction of outcome after curative surgery for colorectal cancer: preoperative haemoglobin, C-reactive protein and albumin[J]. Colorectal Disease, 2018, 20.

21. Haskins I N , Baginsky $M$, Amdur R L , et al. Preoperative hypoalbuminemia is associated with worse outcomes in colon cancer patients[J]. Clinical Nutrition, 2016, 36(5).

22. Zhang $M$, Yang XJ. Effects of a high fat diet on intestinal microbiota and gastrointestinal diseases. World J Gastroenterol. 2016;22(40):8905-8909.

23. Leander $U$, Furst $P$, Vesterberg $K$, et al. Nitrogen sparing effect of Ornicetil in the immediate postoperative state clinical biochemistry and nitrogen balance. Clin Nutr. 1985;4(1):43-51.

24. Matsuda T, Kato H, Suzuki $H$, et al. Within-Day Amino Acid Intakes and Nitrogen Balance in Male Collegiate Swimmers during the General Preparation Phase. Nutrients. 2018;10(11).

25. Garibotto G, Verzola D, Vettore M, et al. The contribution of muscle, kidney, and splanchnic tissues to leucine transamination in humans. Can J Physiol Pharmacol. 2018;96(4):382-387.

26. Gudny Geirsdottir O, Thorsdottir I. Nutritional status of cancer patients in chemotherapy; dietary intake, nitrogen balance and screening. Food Nutr Res. 2008;52.

27. Chiang JM, Chang CJ, Jiang SF, et al. Pre-operative serum albumin level substantially predicts postoperative morbidity and mortality among patients with colorectal cancer who undergo elective colectomy. Eur J Cancer Care (Engl). 2017;26(2).

28. Hu W H, Chen H H, Lee K C , et al. Assessment of the Addition of Hypoalbuminemia to ACS-NSQIP Surgical Risk Calculator in Colorectal Cancer[J]. Medicine, 2016, 95(10):e2999.

29. Lee YP, Chiu CC, Lin TJ, et al. The germ-free mice monocolonization with Bacteroides fragilis improves azoxymethane/dextran sulfate sodium induced colitis-associated colorectal cancer. Immunopharmacol Immunotoxicol. 2019:1-7.

30. Lim So, Gu JM, Kim MS, et al. Epigenetic changes induced by reactive oxygen species in hepatocellular carcinoma: methylation of the E-cadherin promoter. Gastroenterology. 2008;135(6):2128-2140, 2140 e2121-2128.

31. Kim K, Castro EJT, Shim H, et al. Differences Regarding the Molecular Features and Gut Microbiota Between Right and Left Colon Cancer. Ann Coloproctol. 2018;34(6):280-285.

32. Precup G, Vodnar DC. Gut Prevotella as a possible biomarker of diet and its eubiotic versus dysbiotic roles-A comprehensive literature review. Br J Nutr. 2019:1-24.

33. Hiippala K, Kainulainen V, Kalliomaki M, et al. Mucosal Prevalence and Interactions with the Epithelium Indicate Commensalism of Sutterella spp. Front Microbiol. 2016;7:1706.

34. Choi C H R , Bakir I A , Hart A L , et al. Clonal evolution of colorectal cancer in IBD[J]. Nature Reviews Gastroenterology \& Hepatology, 2017, 14(4):218-229. 
35. Paterson MJ, Oh S, Underhill DM. Host-microbe interactions: commensal fungus in the gut. Curr Opin Microbiol. 2017;40:131-137.

36. Glinka P, Nowacka K, Kulesza K, et al. Share of the Saccharomyces genus in the mycobiota of the gastrointestinal tract of oncology patients - potential effects of a fruit-based diet. Ann Parasitol. 2018;64(3):199-202.

37. Shamekhi S, Abdolalizadeh J, Ostadrahimi A, et al. Apoptotic Effect of Saccharomyces cerevisiae on Human Colon Cancer SW480 Cells by Regulation of Akt/NF-kB Signaling Pathway. Probiotics Antimicrob Proteins. 2019.

38. Kaur H, Rudramurthy SM, Mohindra S, et al. Ochroconis humicola Coexisting with Esthesioneuroblastoma: An Incidental Coloniser or Allergen? Mycopathologia. 2014;178(1-2):79-83.

39. Murugesan S, Nirmalkar K, Hoyo-Vadillo C, et al. Gut microbiome production of short-chain fatty acids and obesity in children. Eur J Clin Microbiol Infect Dis. 2018;37(4):621-625.

40. Manrique Vergara D, Gonzalez Sanchez ME. Short chain fatty acids (butyric acid) and intestinal diseases. Nutr Hosp. 2017;34(Suppl 4):58-61.

41. Parada Venegas D, De la Fuente MK, Landskron G, et al. Short Chain Fatty Acids (SCFAs)-Mediated Gut Epithelial and Immune Regulation and Its Relevance for Inflammatory Bowel Diseases. Front Immunol. 2019;10:277.

42. Louis P, Hold GL, Flint HJ. The gut microbiota, bacterial metabolites and colorectal cancer. Nat Rev Microbiol. 2014;12(10):661-672.

43. Machover D, Rossi L, Hamelin J, et al. Effects in cancer cells of the recombinant L-Methionine gamma-lyase from Brevibacterium aurantiacum. Encapsulation in human erythrocytes for sustained L-methionine elimination. J Pharmacol Exp Ther. 2019.

44. Kadochi Y, Mori S, Fujiwara-Tani R, et al. Remodeling of energy metabolism by a ketone body and medium-chain fatty acid suppressed the proliferation of CT26 mouse colon cancer cells. Oncol Lett. 2017;14(1):673-680.

45. Krishnamurthy RV, Suryawanshi YR, Essani K. Nitrogen isotopes provide clues to amino acid metabolism in human colorectal cancer cells. Sci Rep. 2017;7(1):2562.

46. Viana Veloso GG, Franco $\mathrm{OH}$, Ruiter $\mathrm{R}$, et al. Baseline dietary glutamic acid intake and the risk of colorectal cancer: The Rotterdam study. Cancer. 2016;122(6):899-907.

\section{Supplementary Figure Legends}

\section{Figure S1. Bacterial alpha diversity analysis in CRCs with different serum albumin status}

Rarefaction curve is a plot of the number of OTUs as a function of the number of sequencing reads. The flattening of the rarefaction curve indicates that the sequencing depth is appropriate. Panel A shows the Chao1 curve for each sample. Shannon and Simpson index indicates the level of microbial diversity of the sample. Higher Shannon value indicates higher community diversity. Lower Simpson value indicates 
higher community diversity. Panel B and panel C show Shannon curves and Simpson curves, respectively. The box chart describes the average level and variation degree among the three groups.

Figure S2. Fungal alpha diversity analysis in CRCs with different serum albumin status

Panel A shows the Chao1 curve for each sample. Panel B and panel C shows Shannon curves and Simpson curves, respectively. The box chart describes the average level and variation degree among the three groups.

\section{Figures}



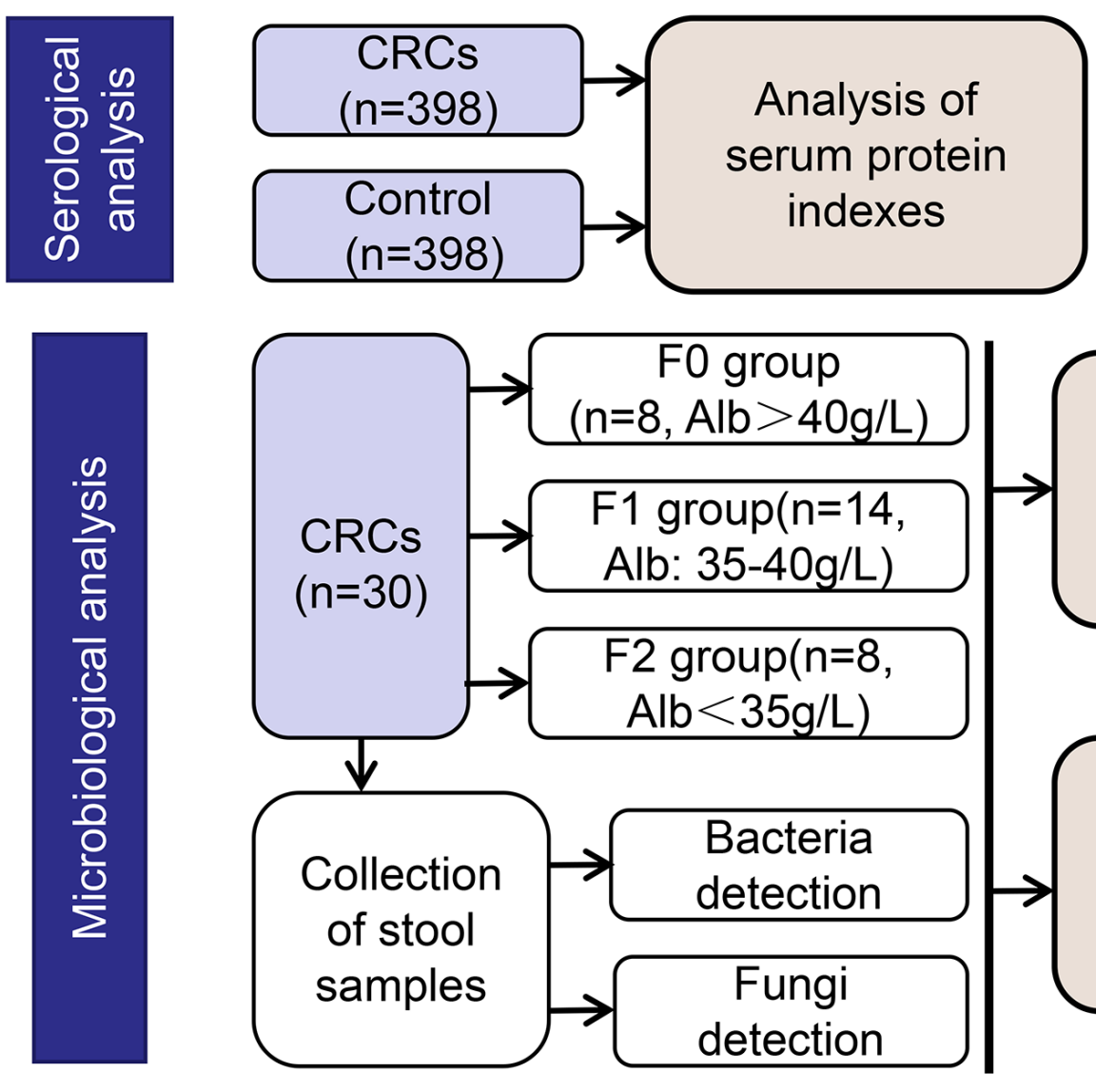

Analysis of fungal diversity and community structure
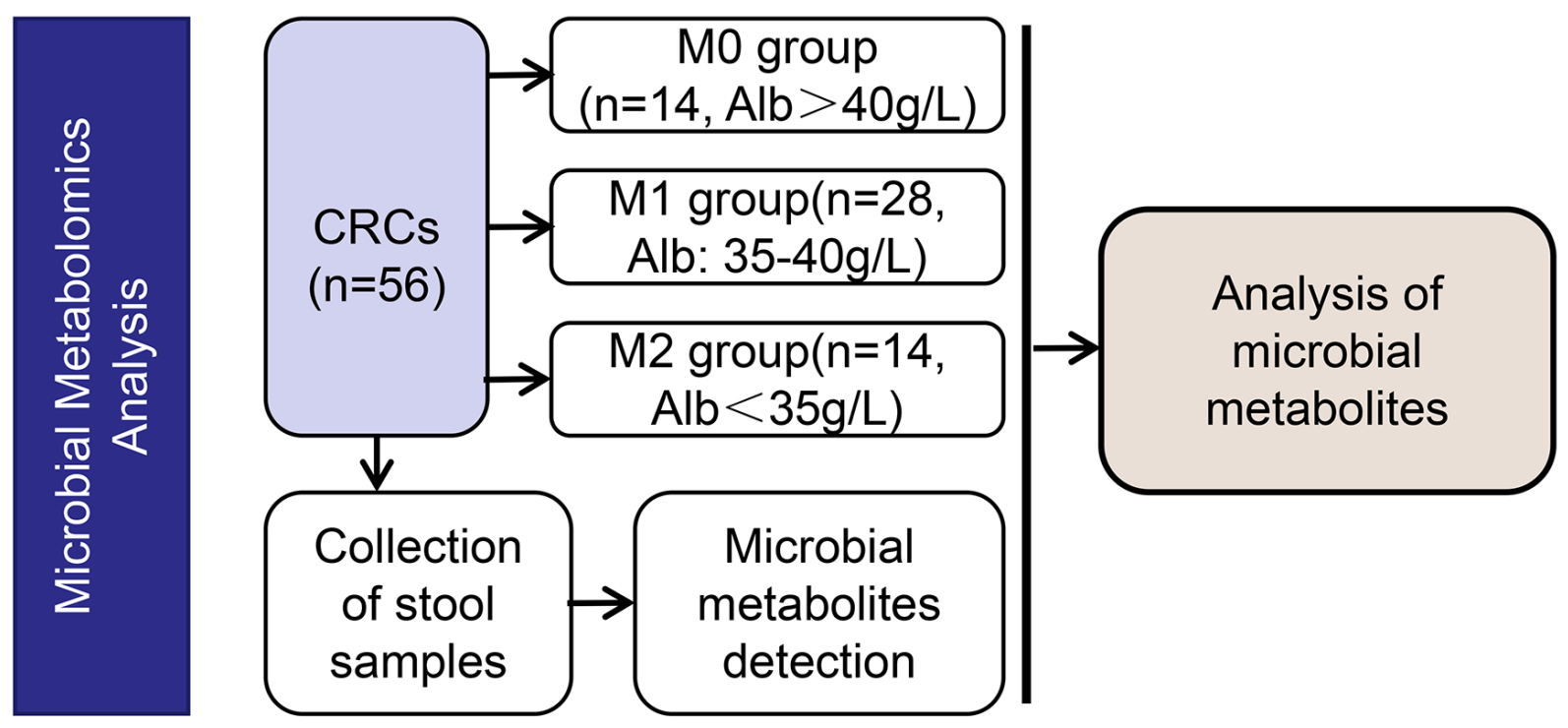

Figure 1

The study design is shown in the figure. General characteristics and serum nutrition-related indexes in CRC patients and age-matched subjects were compared to analyze serum protein levels in CRC patients. The CRC patients can be divided into three groups according to serum protein levels. The gut microbes and microbial metabolites in these three groups were determined and investigated to analyze the correlation between the intestinal microecology and nutritional status. 


\section{genus top8}

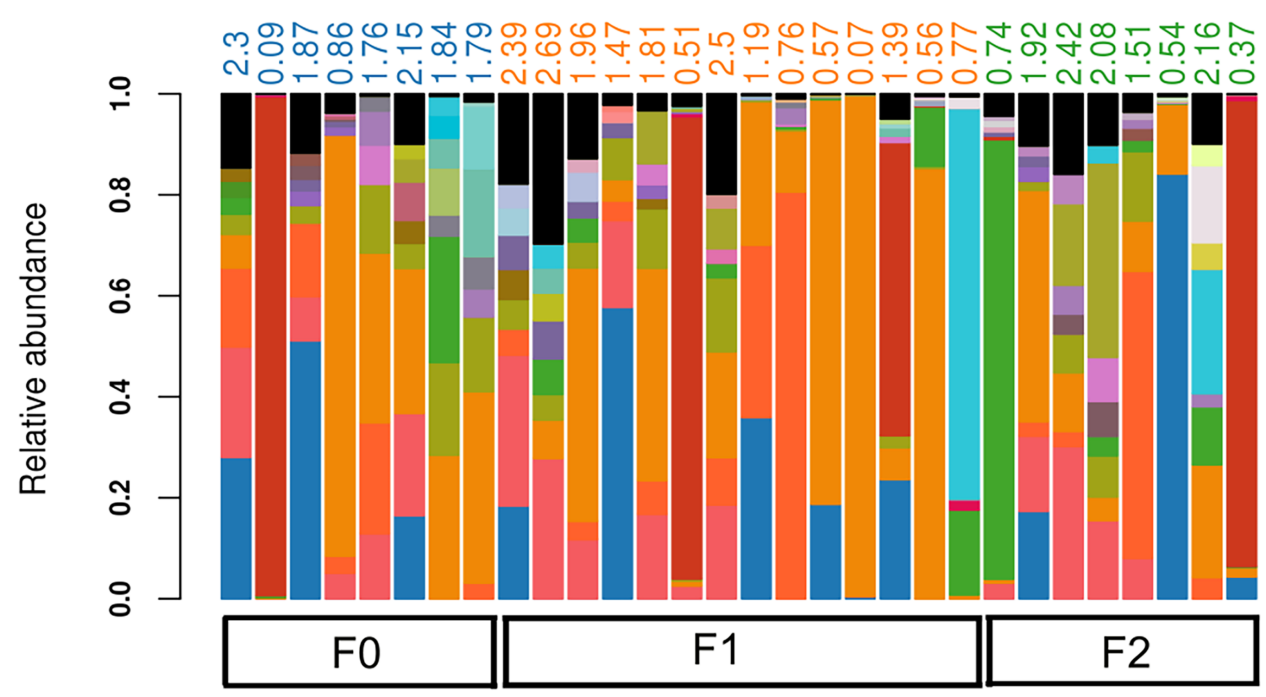

others
f_Incertae_sedis_11
Kodamaea
Wickerhamomyces
Pichia
Gibberella
Auricularia
Geotrichum
Hyalodendriella
Clonostachys
Lodderomyces
Alternaria
Colletotrichum
Aureobasidium
Dioszegia
Acremonium
Yarrowia
Trametes
Polyporales_unidentified_1
f_Saccharomycetaceae
Mucor
Aspergillus
Eurotium
Glomerella
Agaricales_unidentified_1
Hypocreales_unidentified_1
Tremellomycetes_unidentified_1
Agaricomycetes_unidentified_1
Malasseziales_unidentified_1

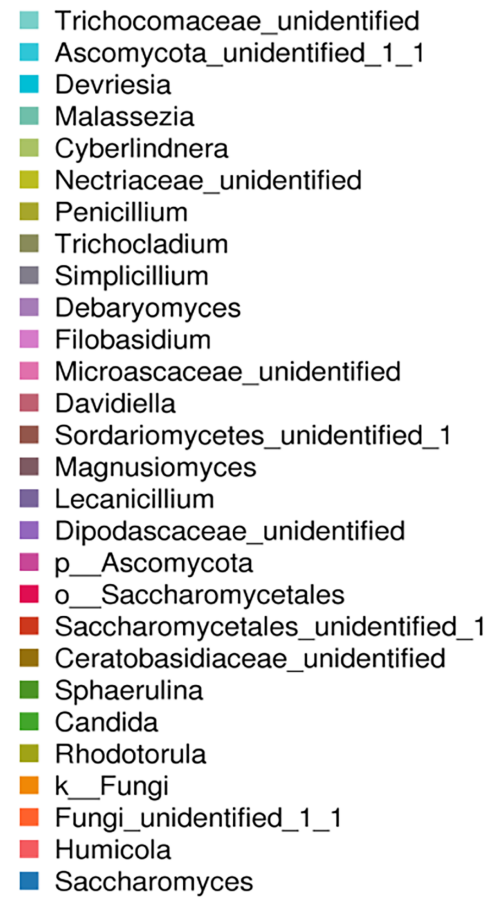

Figure 2

Stool samples were used for the detection of gut microbes. Serum samples from peripheral blood were used for the detection of serum albumin levels. The figure shows the plot of community structure of gut bacteria among the different serum albumin levels. The stacked bar graph shows the relative abundance of the gut bacteria at the genus level for each sample. Considering the visibility of the plot, the relative abundance of bacteria less than $1 \%$ were not included. 


\section{kruskal test: pvalue-0.05}

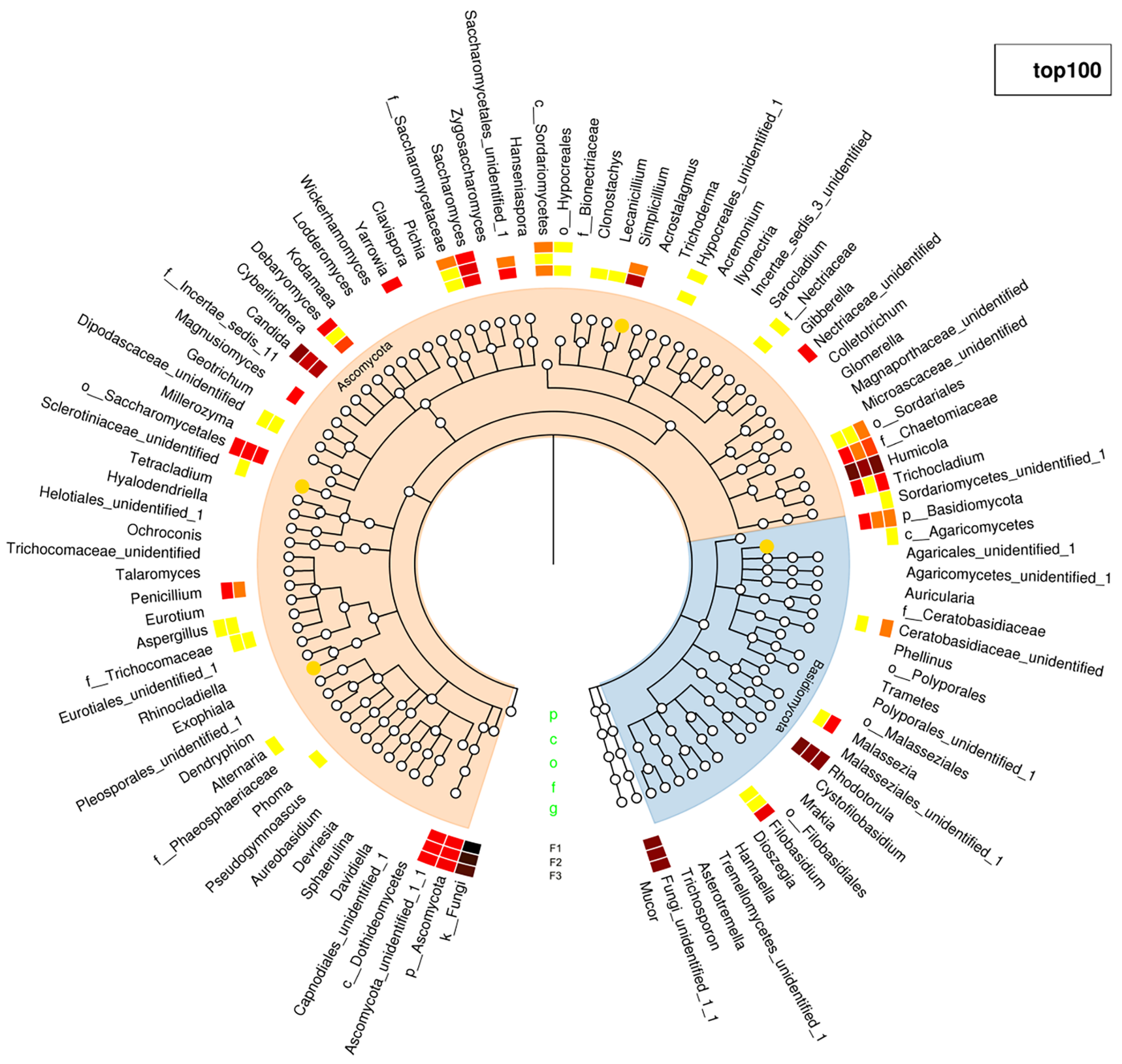

\section{Figure 3}

The taxonomic tree heatmap shows the composition and proportion of bacteria at different taxonomic levels including phylum, class, order, family, and genus. The innermost layer shows the taxonomic tree. The circles from inside to outside represent phylum, class, order, family, and genus. The heatmap(colored rectangles) at the intermediate layer represents the average abundance. The deeper the color, the higher is the species abundance. Orange circles represent statistical difference of species among the three groups 
(Kruskal test, $p<0.05)$. The outermost layer is that the labeled bacteria names. The " $\mathrm{p}_{-}$" "c_" " $\mathrm{o}_{-}$" and " $\mathrm{f}_{-}$" represents "phylum level", "class level", "order level" and "family level", respectively. The default for no similar tags is the genus level.

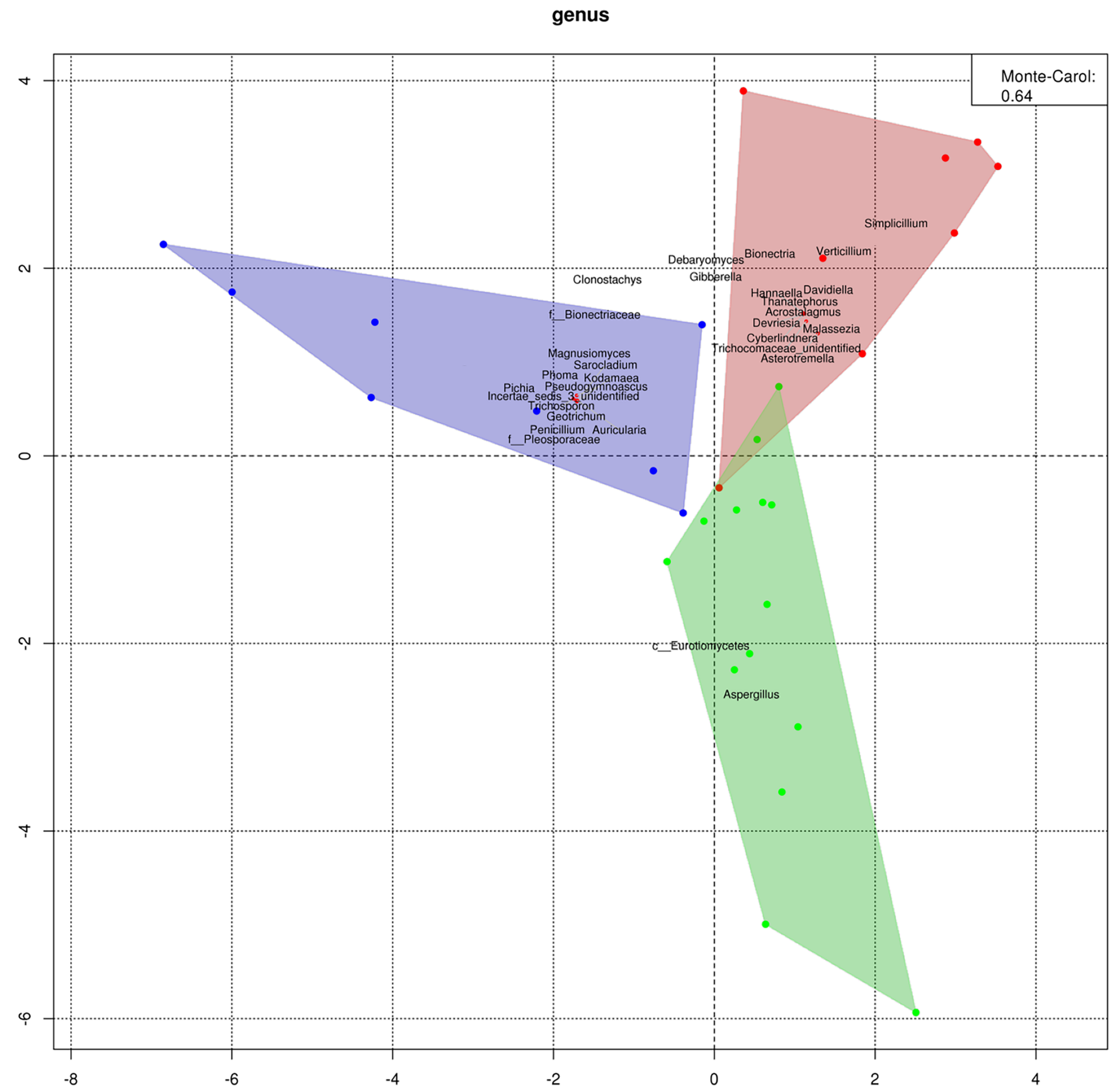

\section{Figure 4}

The enterotype map represents the correlation and contribution of the bacteria to different groups. Red area, green area, and blue area represents F0 group, F1 group, and F2 group, respectively. Each cycle represents a sample. The closer to the center of different colors, the greater the correlation with this group (Monte-Carlo simulation). Some bacteria cannot be annotated to the genus level. "p_" "c_" "o_" and "f_" 
represent "phylum level", "class level", "order level" and "family level", respectively. The default for no similar tags is the genus level.

genus top8

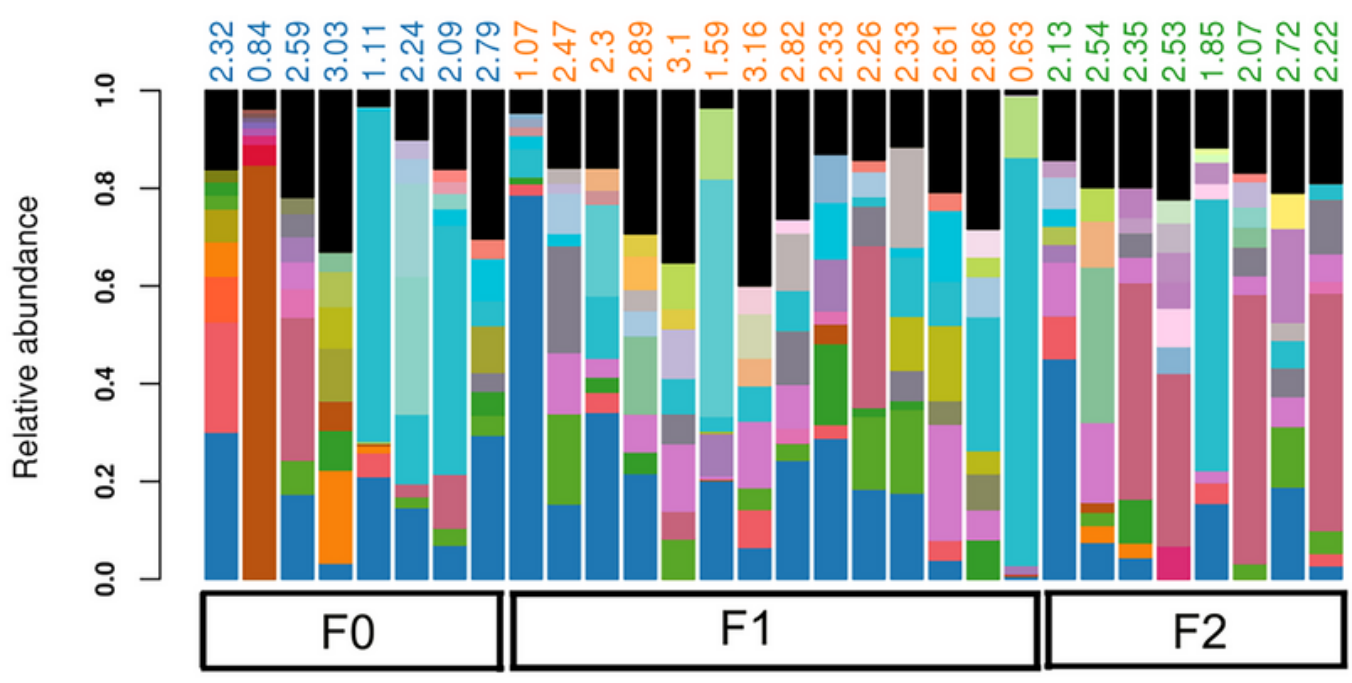

others
Weissella
Solobacterium
Acinetobacter
Peptostreptococcus
Capnocytophaga
Porphyromonas
Alloprevotella
Lactobacillus
Howardella
f_Enterococcaceae
Comamonas
Parvimonas
Catenibacterium
Megasphaera
Enterococcus
O_Clostridiales
Coprococcus
A_Prevotellaceae
Alistipes
Ruminococcus
Fusobacterium
Cetobacterium
Veillonella
Clostridium XVIII
Dorea

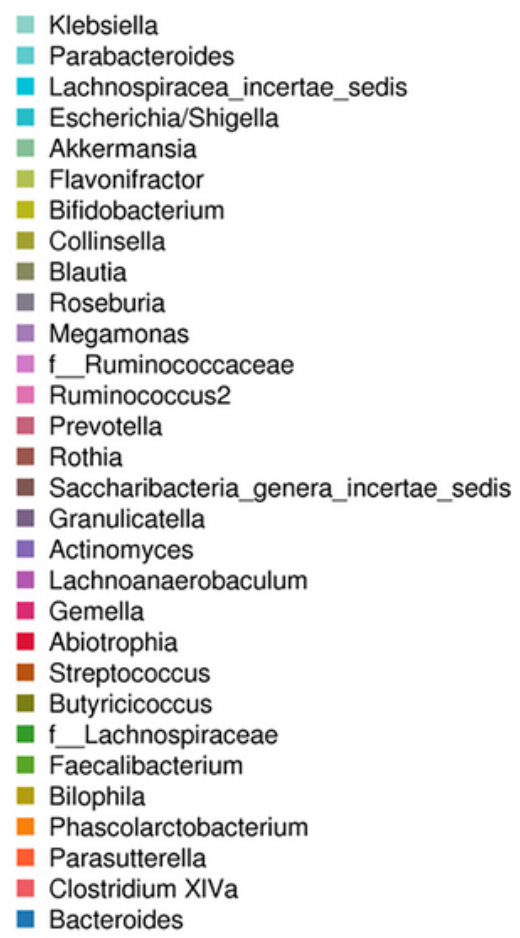

\section{Figure 5}

The figure shows the plot of community structure of gut fungus among the different serum albumin levels. The stacked bar graph shows the relative abundance of the gut fungus at the genus level for each 
sample. Considering the visibility of the plot, the relative abundance of fungus less than $1 \%$ were not included.

\section{kruskal test: pvalue-0.05}

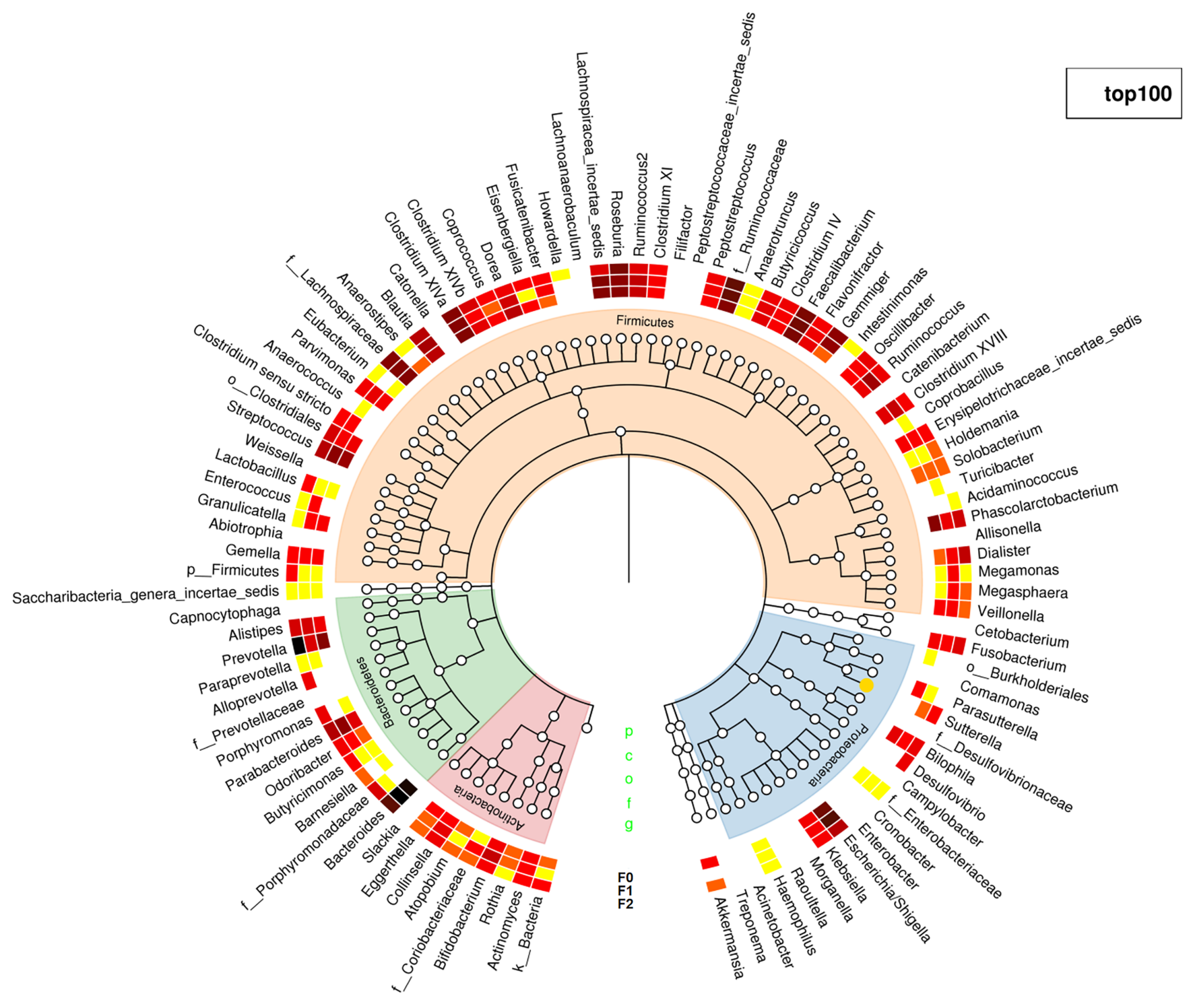

Figure 6

The taxonomic tree heatmap shows the composition and proportion of fungus at different taxonomic levels including phylum, class, order, family, and genus. The innermost layer shows the taxonomic tree. The circles from inside to outside represent phylum, class, order, family, and genus. The heatmap(colored rectangles) at the intermediate layer represents the average abundance. The deeper the color, the higher is the species abundance. Orange circles represent statistical difference of species among the three groups (Kruskal test). The outermost layer is that the labeled bacteria names. " $p_{-}$" " $\mathrm{c}_{-}$" "o_" and "f_" represent "phylum level", "class level", "order level" and "family level", respectively. The default for no similar tags is the genus level. 


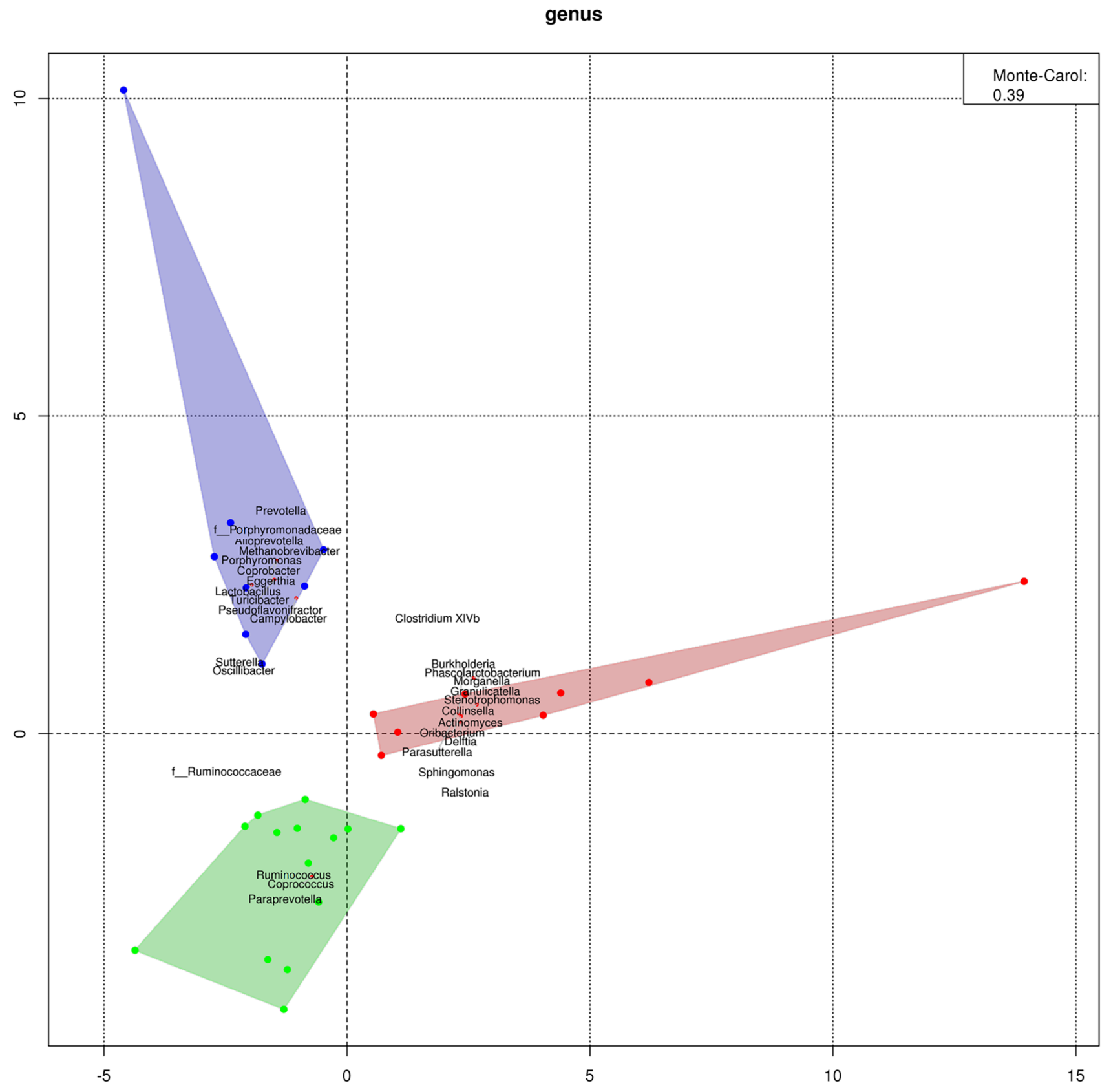

\section{Figure 7}

The enterotype map represents the correlation and contribution of the fungus to different groups. Red area, green area, and blue area represents F0 group, F1 group, and F2 group, respectively. Each cycle represents a sample. The closer to the center of different colors, the greater the correlation with this group (Monte-Carlo simulation). Some bacteria cannot be annotated to the genus level. " $\mathrm{p}_{-}$" "c_" "o_" and "f_" represent "phylum level", "class level", "order level" and "family level", respectively. The default for no similar tags is the genus level. 
A
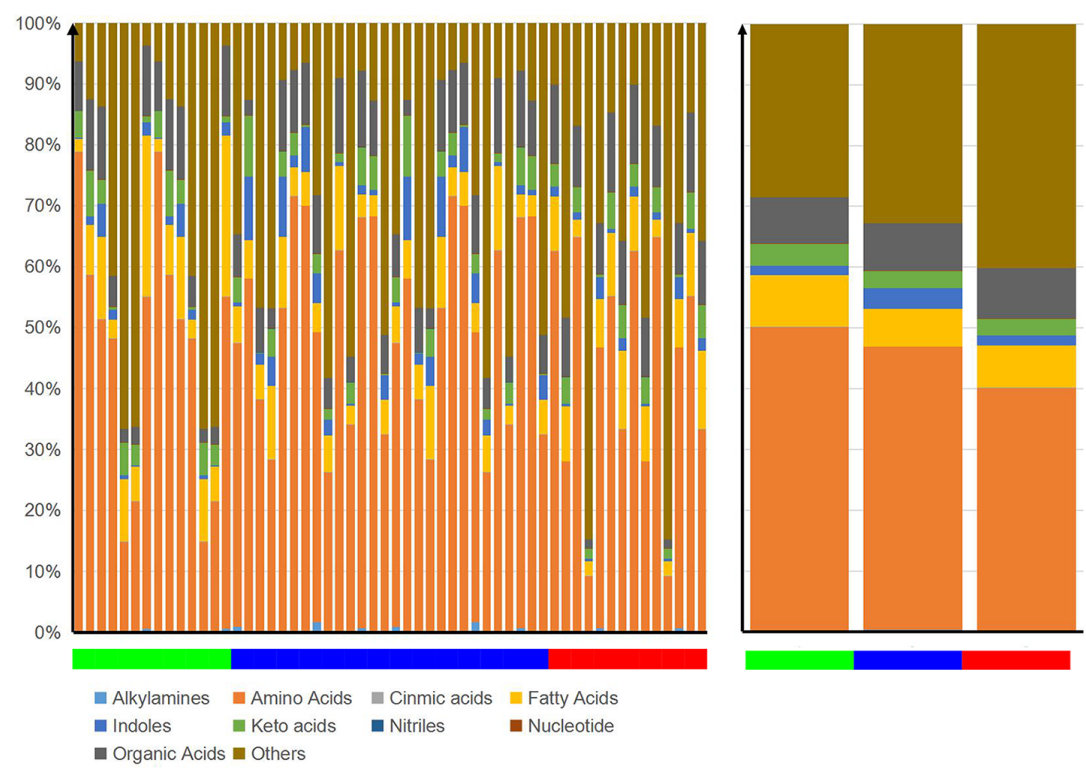

- Organic Acids $\square$ Others

B
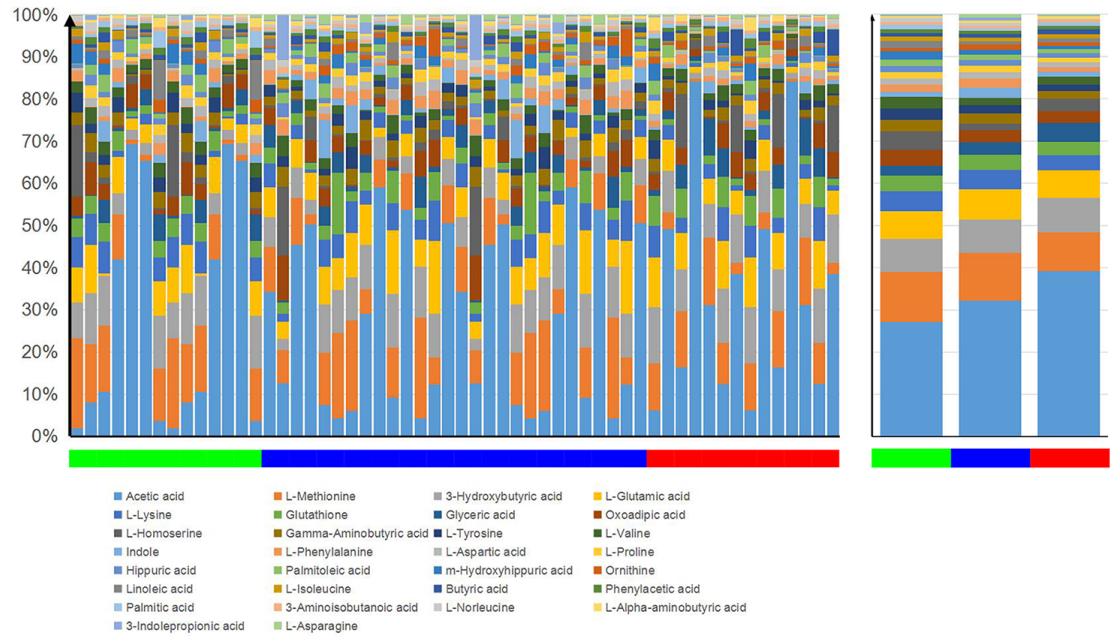

M0 $\square$ M1 M2

\section{Figure 8}

A total of 56 CRC patients were enrolled to determine the gut microbial metabolites. Patients were divided into three groups according to albumin levels. M0, M1 and M2 represent ranges of albumin values of over $40 \mathrm{~g} / \mathrm{L}, 35 \sim 40 \mathrm{~g} / \mathrm{L}$ and below $35 \mathrm{~g} / \mathrm{L}$, respectively. In total, 124 microbial metabolites in 10 classes extracted from stool samples of CRC patients were quantified. The panel A in Figure 4 shows the 
proportion of gut microbial metabolites at class level. The panel B in Figure 4 shows the proportion of gut microbial metabolites in the absolute quantitative top 30.

A

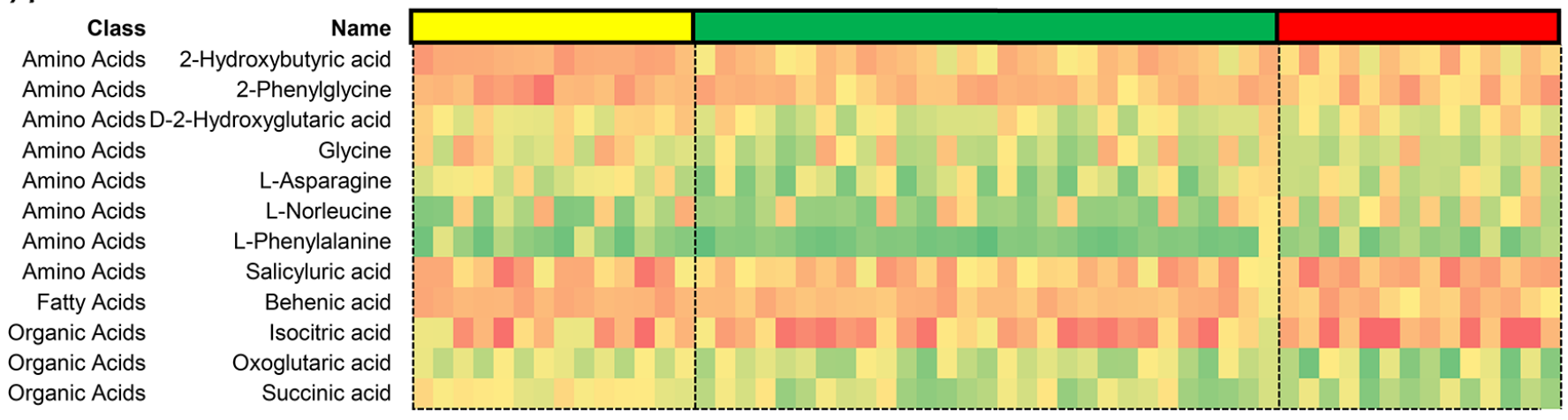

B

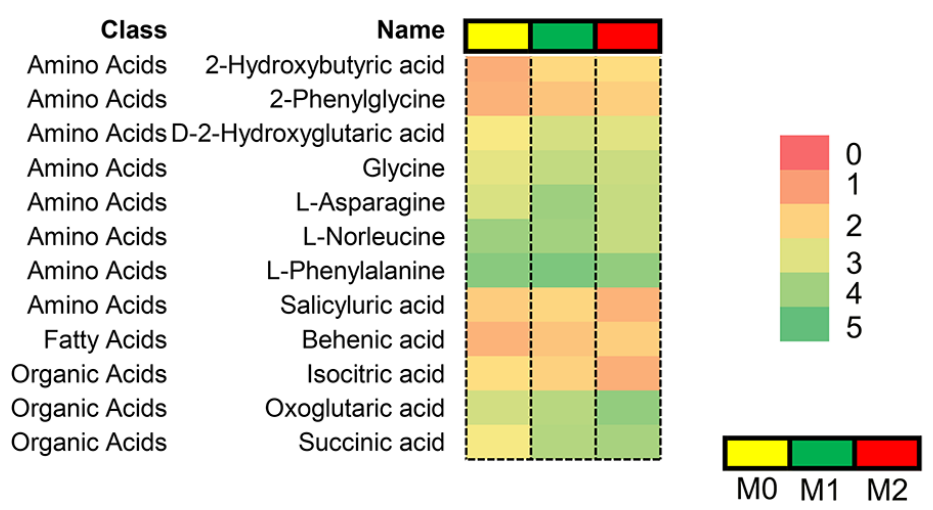

Figure 9

The GC/TOFMS analysis was used to determine the amount of microbial metabolites per milliliter of the stool samples from $56 \mathrm{CRC}$ patients. Patients were divided into three groups according to albumin levels. $\mathrm{M0}, \mathrm{M} 1$ and $\mathrm{M} 2$ represent ranges of albumin values of over $40 \mathrm{~g} / \mathrm{L}, 35 \sim 40 \mathrm{~g} / \mathrm{L}$ and below $35 \mathrm{~g} / \mathrm{L}$, respectively. The figure shows the gut microbial metabolites with statistically significant differences among the three groups (Kruskal test). The logarithm base 10 of the sample concentration $(\mathrm{ng} / \mathrm{ml})$ was used to generate a heat map. Panel A and panel B show the gut microbial metabolites from each sample and each group, respectively.

\section{Supplementary Files}

This is a list of supplementary files associated with this preprint. Click to download.

- Supplementalmaterial1.doc

- SupplementmaterialfigureS1.tif 
- SupplementmaterialfigureS2.tif

- SupplementmaterialtableS1.docx 